\title{
A study of mixing in coherent vortices using braiding factors
}

\author{
M. R. Turner ${ }^{1} 2$ \& M. A. Berger \\ Mathematics Research Institute, \\ College of Engineering, Mathematics and Physical Sciences, \\ University of Exeter, \\ Exeter EX4 4QF, U.K.
}

\begin{abstract}
This paper studies the use of braiding fluid particles to quantify the amount of mixing within a fluid flow. We analyze the pros and cons of braid methods by considering the motion of three or more fluid particles in a coherent vortex structure. The relative motions of the particles, as seen in a space-time diagram, produces a braid pattern, which is correlated with mixing and measured by the braiding factor.

The flow we consider is a Gaussian vortex within a rotating strain field which generates cat's eyes in the vortex. We also consider a modified version of this strain field which contains a resonance frequency effect that produces multiple sets of cat's eyes at different radii. As the thickness of the cat's eyes increase they interact with one another and produce complex Lagrangian motion in the flow which increases the braiding of particles, hence implying more mixing within the vortex.

It is found that calculating the braiding factor using only three fluid particles gives useful information about the flow, but only if all three particles lie in the same region of the flow, i.e. this gives good local information. We find that we only require one of the three particles to trace a chaotic path to give an exponentially growing braiding factor. i.e. a non-zero 'braiding exponent'. A modified braiding exponent is also introduced which removes the spurious effects caused by the rotation of the fluid.

This analysis is extended to a more global approach by using multiple fluid particles that span larger regions of the fluid. Using these global results we compare the braiding within a viscously spreading Gaussian vortex in the above strain fields, where the flow is determined both kinematically and dynamically. We show that the dynamic feedback of the strain field onto the flow field reduces the overall amount of braiding of the fluid particles.
\end{abstract}

1 Current address: Sir Harry Ricardo Laboratories, School of Computing, Engineering and Mathematics, Cockcroft building, University of Brighton, Lewes Road, Brighton. BN2 4GJ. UK

2 Corresponding author: M.R.Turner@brighton.ac.uk 


\section{Introduction}

Various techniques have been used to measure the amount of mixing within chaotic flows, such as using the effective diffusion (Shuckburgh and Haynes, 2003; Turner et al., 2008, 2009b) and Lyapunov exponents (Wolf et al., 1985; Lichtenberg and Lieberman, 1992; Pierrehumbert and Yang, 1993) to name but two. One of the latest techniques studied for such a purpose is that of particle braiding (Boyland et al., 2000; Kin and Sakajo, 2005; Thiffeault, 2005, 2010). Braiding uses the trajectories of particles within the flow to give a measure of their entanglement, and hence how chaotic the flow is. The measure of entanglement is known as the 'braiding exponent' (or the braid's topological entropy) and is a lower bound on the rate of stretching of material lines (Boyland et al., 2000) which is related to the traditional finite-time Lyapunov exponents (Wolf et al., 1985); the largest positive Lyapunov exponent provides a measure of chaotic motion in advection (Aref, 1984). The main appeal of the braiding exponent compared to the Lyapunov exponent is the ease with which it can be computed, even from crude data sets, without knowledge of the underlying fluid flow (Kin and Sakajo, 2005; Thiffeault, 2005, 2010). The braiding exponent is also more sensitive to global properties of the flow compared to Lyapunov exponents. The fact that the braiding exponent can be easily computed makes it appealing to use on fluid dynamics problems, because mixing properties of particular flows can be inferred by solely considering the paths of individual particles such as tracer particles in experiments. So far braiding has mainly been used to quantify the mixing properties of idealized dynamical systems, such as free point vortices (Boyland et al., 2000), fixed blinking vortices (Kin and Sakajo, 2005; Thiffeault, 2005) or rod stirring devices etc. (Finn et al., 2003; Vikhansky, 2004; Binder and Cox, 2008; Thiffeault et al., 2008). Thiffeault (2010) then applied the braiding technique to a set of sea float data to imply information about the complex motion of the Labrador sea (Davis, 2004), however this study did not thoroughly investigate the use of braiding on a 'real' flow because the flow field in the Labrador sea was not known explicitly, so no connections could be made between the braiding results and the motion of the fluid in real space. Therefore, the aim of this work is to use this technique on another real flow problem, consisting of a coherent vortex structure in a rotating strain field, to examine the pros and cons of this diagnostic and link the braiding results to the motion of the fluid particles in real space. The main reason for conducting this study is because braiding can be used to infer information about a fluid flow, such as the Labrador sea data, without requiring knowledge of the flow field itself, which could aid experiments where tracer particles are placed in the flow, but the flow field itself is unknown. 
We study the evolution of fluid particle trajectories within the two-dimensional axisymmetric Gaussian vorticity field

$$
\omega_{0}(r, t)=\frac{1}{4 \pi\left(L^{2}+t R^{-1}\right)} \exp \left(-\frac{r^{2}}{4\left(L^{2}+t R^{-1}\right)}\right)
$$

where $L$ is some initial width of the vortex and $R=\Gamma / \nu$ is the Reynolds number based on the total circulation $\Gamma$. This vortex is placed in an irrotational external strain field $\psi_{\text {ext }}$ which takes one of the following two forms: either $\psi_{\text {ext }}$ is a steadily rotating strain field with angular velocity $\alpha_{\text {ext }}$ which generates cat's eyes at a single radius $r_{\text {ext }}$ within the vortex (Turner and Gilbert, 2007) or $\psi_{\text {ext }}$ is a rotating strain field with multiple resonant frequencies that generates multiple cat's eyes which, if wide enough, overlap and produce regions of complex Lagrangian motion. We examine the effect of both these strain fields on a steady vortex (1.1) (with $R=\infty$ ) using a kinematic integral flow model. This approach does not incorporate any feedback of the strain field onto the basic vortex profile (Bassom and Gilbert, 1999; Turner and Gilbert, 2007). This part of the study will provide a flow field which will allow us to examine the limitations of the braiding method and determine in which situations this technique will be useful.

Once the properties of the braiding method have been established for the steady kinematic flow model, we use it to compare the mixing properties of a kinematic flow and a dynamical flow model. For the strain field which generates one set of cat's eyes in the vortex at $r=r_{\text {ext }}$, the dynamical aspects of the flow cause the azimuthal averaged profile, $\omega_{0}(r, t)$, to flatten (Turner and Gilbert, 2007) which leads to enhanced spreading and mixing within the vortex. However, it was also observed that this flattening leads to a resonance effect occurring within the vortex which produces further mixing and spreading (Turner and Gilbert, 2009). The resonance effect occurs because the vortex profile (1.1) has a particular radius $r_{\text {cat }}(t)$ at which inviscid cat's eyes of infinitesimal width can exist without the application of an external strain field (Le Dizès, 2000). When the radius $r_{\text {cat }}(t)$ becomes close to the externally forced radius $r_{\text {ext }}(t)$ there is a rapid feedback in the vorticity field, producing large-amplitude, non-axisymmetric components of the vorticity field. When we consider the case $R=10^{4}$ with an initial vortex profile close to a point vortex, we find that the value of $r_{\text {cat }}(0) \approx 0$ and $r_{\text {ext }}(0)$ lies outside the vortex. Then, as the vortex spreads, $r_{\text {cat }}(t)$ moves radially outwards (i.e. $r_{\text {cat }}(t)$ increases) while $r_{\text {ext }}(t)$ moves radially inwards $\left(r_{\text {ext }}(t)\right.$ decreases $)$, until $r_{\text {cat }} \approx r_{\text {ext }}$ and the resonant response occurs. This resonant response does not occur in the kinematic flow model because there is no dynamical feed back onto the flow field to generate non-axisymmetric components of vorticity. Bassom and Gilbert (1999) found that applying a white noise random strain field to (1.1) suppressed the amount of mixing in the core of the vortex. This was extended by Turner et al. (2009a), in a nonlinear study, who found that at the edge of 
the vortex is a 'vorticity staircase' which forms a barrier with the core that stops vorticity flowing out and creates a 'surf zone' of well mixed fluid around the core. In this study we expect to see an enhanced mixing effect similar to this for the multiple cat's eye strain field as this multiple frequency forcing is akin to a random strain, which contains all possible frequencies. The approach studied in Turner and Gilbert (2009) is an Eulerian approach which generated results with a visual appearance of mixing, therefore the present study quantifies this further by focusing on the Lagrangian aspects of the flow.

The content of the paper is laid out as follows. In $\S 2$ we formulate the kinematic integral flow model, while in $\S 3$ we discuss how the braiding results will be calculated. These results are used in $\S 4$ where we examine the effect of the two strain fields on the steady Gaussian vortex (1.1). In $\S 5$ we extend these results to an unsteady viscously spreading vortex and compare the kinematic flow model results with the dynamical flow simulations. Our conclusions and discussions are given in $\S 6$.

\section{Formulation}

We study the evolution of a two-dimensional axisymmetric vortex with vorticity profile $\omega_{0}^{*}\left(r^{*}, t^{*}\right)$ in an externally imposed irrotational strain field with stream function $\psi_{\text {ext }}^{*}\left(r^{*}, \theta, t^{*}\right)$ where $\left(r^{*}, \theta\right)$ are plane polar coordinates and the stars represent dimensional variables. The irrotational strain field $\psi_{\text {ext }}^{*}\left(r^{*}, \theta, t^{*}\right)$ takes the form

$$
\psi_{\text {ext }}^{*}\left(r^{*}, \theta, t^{*}\right)=q^{*}\left(t^{*}\right) r^{* m} e^{i m \theta}+\text { complex conjugate, }
$$

and corresponds to a strain field with an $m$-fold symmetry in the $\theta$-direction and a time dependent part $q^{*}\left(t^{*}\right)$. The precise form of $q^{*}\left(t^{*}\right)$ will be given later, but we assume that it has some amplitude $\hat{A}$ and that $t^{*}$ appears in the combination $\alpha_{\text {ext }} t^{*}$, where $\alpha_{\text {ext }}$ is the angular velocity of the strain field. Therefore we introduce the non-dimensional variables

$$
t=\alpha_{\text {ext }} t^{*}, \quad r=\left(\alpha_{\text {ext }} / \Gamma\right)^{1 / 2} r^{*},
$$

where $\Gamma$ is the total circulation in the vortex at $t=0$. Therefore, in nondimensional variables, the strain field (2.1) becomes

$$
\psi_{\text {ext }}(r, \theta, t)=q(t) r^{m} e^{i m \theta}+\text { complex conjugate, }
$$

where the amplitude of $q(t)$ is now

$$
A=\frac{\hat{A}}{\alpha_{\mathrm{ext}}}\left(\frac{\alpha_{\mathrm{ext}}}{\Gamma}\right)^{2-m},
$$


and in the case where $m=2$, corresponds solely to the ratio of dimensional strain amplitude to the dimensional strain angular velocity.

The major part of this work focuses on the kinematic flow model where the total stream function of the flow is given by $\psi(r, \theta, t)=\psi_{0}(r, t)+\psi_{\text {ext }}(r, \theta, t)$, and $\psi_{0}(r, t)$ is found by solving $\omega_{0}=-\nabla^{2} \psi_{0}$ for the vortex (1.1). Therefore we can then follow the evolution of an individual fluid particle by simultaneously solving the equations

$$
\frac{d r}{d t}=\frac{1}{r} \frac{\partial \psi}{\partial \theta}, \quad \frac{d \theta}{d t}=-\frac{1}{r} \frac{\partial \psi}{\partial r}
$$

In this kinematic flow approach, the initial vorticity field is not changed by the strain field, while in the dynamical flow approach, there would be a nonlinear feed back from the velocity field onto the vorticity field through the relation $\omega=-\nabla^{2} \psi$ (Turner and Gilbert, 2009). The effect of such a feedback and its comparison to the kinematic flow approach is examined in $§ 5.2$. Although this dynamical flow approach is more realistic in flows with large forcing amplitudes $A$, the kinematic flow simulations are very useful because they contain many of the key features seen in the fully dynamical simulations and are much easier to simulate and understand.

In this paper we focus our attention on the viscously spreading vorticity field (1.1) which corresponds to the flow field with stream function

$$
\psi_{0}(r, t)=-\frac{1}{2 \pi} \int_{0}^{r} \frac{1}{s}\left(1-\exp \left(-\frac{s^{2}}{4\left(L^{2}+t R^{-1}\right)}\right)\right) d s,
$$

where the parameter $L$ gives the width of the Gaussian vortex at $t=0$. We set the value $L=0.04$ in this study so that comparisons with the work of Turner and Gilbert (2009), with $R=10^{4}$, can be made.

The time dependent part of the strain field $q(t)$ from (2.2) will take either of the two forms

$$
q_{1}(t)=A e^{-i m t}
$$

or

$$
q_{2}(t)=\frac{A}{\beta}\left(e^{\beta e^{-i m t}}-1\right),
$$

where $A$ is the amplitude (2.3) of the forcing and $\beta$ is a nonlinearity parameter. The stream function $\psi_{\text {ext }}$ with $q_{1}(t)$ (which we denote as $\psi_{\text {ext1 }}$ ) rotates with angular velocity of magnitude unity and generates cat's eyes in the co-rotating stream function $\psi_{\text {co }}=\psi_{0}+\psi_{\text {ext } 1}+r^{2} / 2$ at the radius $r_{\text {ext }}$. The value of $r_{\text {ext }}$ is found by solving $\alpha\left(r_{\text {ext }}, t\right)=1$ for a particular value of $t$, where

$$
\alpha(r, t)=\frac{1}{r^{2}} \int_{0}^{r} \omega_{0}(s, t) s d s=\frac{1}{2 \pi r^{2}}\left(1-\exp \left(-\frac{r^{2}}{4\left(L^{2}+t R^{-1}\right)}\right)\right)
$$


is the angular velocity of the vortex (1.1). From (1.1) we can see that the vortex spreads on an $O(R)$ timescale, and as the vortex spreads the value of $r_{\text {ext }}$ found by solving $\alpha\left(r_{\text {ext }}, t\right)=1$ reduces, until eventually $r_{\text {ext }}=0$ and the cat's eyes vanish from the flow field. It can be shown that the cat's eyes will vanish at $t=t_{\text {van }}$ which satisfies

$$
t_{\mathrm{van}}=R\left((8 \pi)^{-1}-L^{2}\right)
$$

which for the case $L=0.04$ occurs at $t_{\text {van }} \approx 382$. For more information on this process see $\S 3$ of Turner and Gilbert (2009). The other strain field $\psi_{\text {ext }}$ with $q_{2}(t)$ (denoted by $\psi_{\text {ext2}}$ ) is related to $\psi_{\text {ext1 }}$, because in the limit $\beta \rightarrow 0$ both strain fields are identical and only excite one harmonic frequency in the vortex. However when $\beta \neq 0$ then $\psi_{\text {ext2 }}$ excites cat's eyes at multiple radii which, when wide enough, interact with one another and produce chaos within the velocity field through resonance overlap (Lichtenberg and Lieberman, 1992). We expect this chaos to produce large amounts of mixing within the vortex, so we follow trajectories of individual particles placed in the flow field and use this information to analyse the flow using braiding theory (Boyland et al., 2000). For the results in this paper we set the nonlinearity parameter $\beta=1$ as this gives an interesting flow pattern to study. We also solely consider the case $m=2$, as $m=1$ is a pure translation mode, which is of no dynamical interest, so $m=2$ is the first non-trivial solution to the dynamical flow problem, with which we make comparisons in $\S 5$. Although we only consider $m=2$ here, we expect quantitatively similar results for $m>2$.

\section{Braid diagnostics}

\subsection{Acquiring braid patterns from the flow history}

To calculate the braiding information we place $N$ particles in the flow field at time $t=0$. We then follow their motions. As they move in two-dimensional space and time, a three-dimensional space-time diagram can be used to record the history of their motions. The history of each individual particle is then captured as a curve in this diagram; the set of $N$ curves can intertwine in some complex fashion, forming a braid. We choose initial points for the particles at the radial values

$$
r_{i}=\frac{1}{10}+\frac{(i-1)}{2(N-1)}, \quad i=1, \ldots N
$$

with the corresponding $\theta_{i}$ values distributed randomly in the range $[0,2 \pi]$ using a uniform distribution. We analyze the braiding in the flow using at least $N=3$ particles. In general, if we follow $N$ particles, we can either 
examine the complete braid of $N$ curves; or choose subsets of $p$ curves (where $3 \leq p<N)$, to obtain a distribution of braids.

The procedure we use to examine the braiding evolution of the particles is an extension of that used by Boyland et al. (2003), Vikhansky (2003), and Thiffeault (2005). The main aim of the method is to relate the motion of the particles to elements of the braid group. Thiffeault (2010) uses an alternative method to calculate the braiding exponent based on the method of Moussafir (2006). This method is more difficult to implement numerically than the one we use in this paper, but it has been shown to give a more accurate braiding exponent, while the method in Thiffeault (2005) only gives a lower bound for this value. However, we expect the results of each method to agree qualitatively with one another, and this is sufficient for determining the usefulness or otherwise of using braiding on the flows considered here. If more accurate values of the braiding exponent are required then the method in Thiffeault (2010) should be implemented.

Initially we project the positions of the particles onto the $x$-axis (this choice is purely arbitrary and any reference line can be used), and we label the particles 1 to $p$ from the smallest $x$ value to the largest. A crossing of two particles occurs when they change positions on the $x$-axis. The interchange can occur as an 'over' or 'under' braid, which refers to a clockwise or counterclockwise braid respectively. Thus we define $\sigma_{i}$ as the clockwise interchange of particles $i$ and $i+1$, and $\sigma_{i}^{-1}$ as the counterclockwise interchange, see figure 1. By naming all the such interchanges in this way, we note that the elements $\sigma_{i}$ for $i=1,2, \ldots, p-1$ are the generators of the Artin braid group on $p$ strings (Murasugi, 1996).

(a)

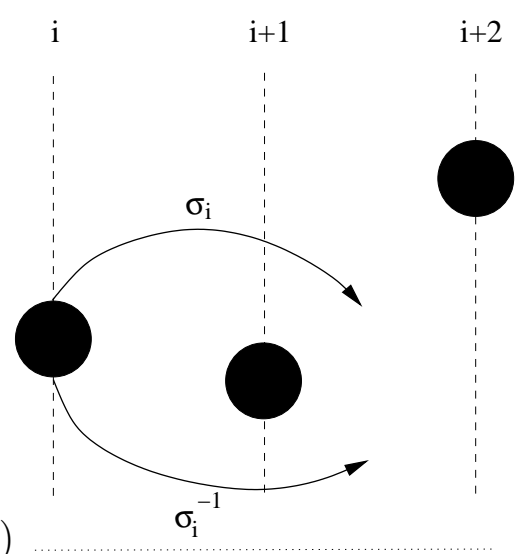

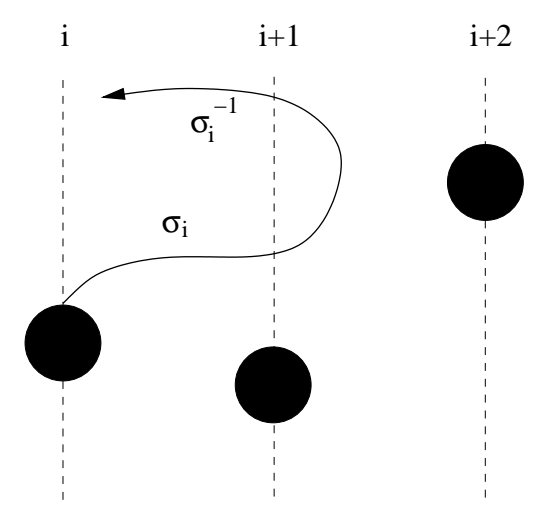

(b)

Fig. 1. Diagram showing the possible detected crossings in the flow: (a) Two possible partial crossings, each of which are associated to different braid group elements; (b) Two crossings that give no net braid. The reference line is given by the dotted line. 


\subsection{The braiding factor}

We represent each element of the braid group by matrices given by the Burau representation (Burau, 1936; Jones, 1987) of the $p$-braid group. Each element of the $p$-braid group is represented by the $(p-1) \times(p-1)$ matrix defined by

$$
\left[\sigma_{i}\right]_{k l}=\delta_{k l}+\delta_{k, i-1} \delta_{l i}-\delta_{k, i+1} \delta_{l i}
$$

with inverses

$$
\left[\sigma_{i}^{-1}\right]_{k l}=\delta_{k l}-\delta_{k, i-1} \delta_{l i}+\delta_{k, i+1} \delta_{l i}
$$

where $i, k, l=1, \ldots, p-1$ and we set $\delta_{k, 0}=\delta_{k, p}=0$. Note that here $\sigma_{i}$ represents both the interchange of two particles and its matrix in the Burau representation of the Artin braid group. Each of these matrices has determinant 1, and satisfies the 'physical braid' conditions (Murasugi, 1996)

$$
\sigma_{i} \sigma_{j}=\sigma_{j} \sigma_{i} \quad \text { for } \quad|i-j| \geq 2 \text {, }
$$

and

$$
\sigma_{i} \sigma_{i+1} \sigma_{i}=\sigma_{i+1} \sigma_{i} \sigma_{i+1}
$$

For the case when $p=3$, the two braid group elements are represented by the $2 \times 2$ matrices

$$
\sigma_{1}=\left[\begin{array}{cc}
1 & -1 \\
0 & 1
\end{array}\right] \quad \sigma_{2}=\left[\begin{array}{ll}
1 & 0 \\
1 & 1
\end{array}\right],
$$

and the physical braid condition reduces to

$$
\Delta=\sigma_{1} \sigma_{2} \sigma_{1}=\sigma_{2} \sigma_{1} \sigma_{2}
$$

where (3.3) defines $\Delta$.

As crossings are detected, due to the motion of the particles, we keep a running total of the product of these matrices. We denote the product of $P(t)$ matrices $(P(t)$ crossings after time $t)$ as

$$
M^{(P)}=\sigma^{(P)} \sigma^{(P-1)} \cdots \sigma^{(2)} \sigma^{(1)}
$$

where $\sigma^{(j)} \in\left\{\sigma_{i}, \sigma_{i}^{-1}: i=1,2, \ldots, p-1\right\}$. From this matrix we can define the braiding factor

$$
\lambda(t)=\max \left(\left\{\mid \text { eigenvalues of } M^{(P)} \mid\right\}\right),
$$

at time $t$.

The topological entropy $\mu(t)$ is the logarithm of this quantity. The topological entropy can be interpreted as follows: if we repeat the braid pattern $M^{(P)} N$ 
times, then at least one material line drawn in the fluid medium will lengthen by a factor

$$
(\lambda(t))^{N}=e^{\mu(t) N}
$$

As we gather longer and longer histories of the flow, the braid pattern increases in length, with a corresponding increase in maximum eigenvalue. For complex flows this increase is exponential in time. Thus we can define the finite time braiding exponent as

$$
\Lambda(t)=\frac{1}{t} \ln |\lambda(t)|
$$

and in the limit $t \rightarrow \infty$ we have the braiding exponent defined by Thiffeault (2005). When this exponent is positive then we say that the braid sequence exhibits 'topological chaos'. We note that the quantity $\Lambda(t)$ is a function of the number of particle tracers $p$.

In $\S 4$ and $\S 5$ when we use the 3 -braid braiding factor to analyze local regions of the flow, we define the trio of particles starting from radii $r_{a}, r_{b}$ and $r_{c}$ in (3.1) as the trio of particles $(a, b, c)$. We also use the $p$-braid with $p>3$ to give us a view of the braiding properties, and hence mixing properties, of the whole flow field.

\subsection{Correcting the braiding factor}

Thiffeault (2005) observes that the braiding factor can often exhibit a highly erratic behaviour. We also see this behaviour in our simulations (see figure $5(\mathrm{a})$ ) where the braiding factor suddenly returns to unity at regular intervals. In this section we explain why this happens and define a modification of the braiding factor (3.13) which removes this behaviour.

The source of the difficulty with braiding factors lies with the effect of uniform rotations. A uniform rotation of a flow should not lead to any mixing, as material particles keep their relative positions. Unfortunately, the braiding factor is not completely immune to the influence of rotations, as particles still interchange position on the reference line and so $M^{(P)}$ gets multiplied by another interchange matrix.

For the three-braid system, a half turn of the vortex results in the braid $\Delta$ defined in (3.3); a complete turn results in $\Delta^{2}$. The braid $\Delta^{2}$ commutes with any other braid $B$, i.e. $\Delta^{2} B=B \Delta^{2}$. We note that complete turns do not cause any difficulties, as $\Delta^{2}$ has no effect on the braiding factor: the Burau matrix is

$$
\Delta^{2}=\left[\begin{array}{cc}
-1 & 0 \\
0 & -1
\end{array}\right]
$$


thus the matrix representing a braid $\Delta^{2} B$ has the same eigenvalues (in magnitude) as the matrix representing $B$.

Half turns, however, have an interesting effect. They almost commute with other braid elements, in the sense that

$$
\begin{array}{ll}
\Delta \sigma_{1}=\sigma_{2} \Delta ; & \Delta \sigma_{1}^{-1}=\sigma_{2}^{-1} \Delta ; \\
\Delta \sigma_{2}=\sigma_{1} \Delta ; & \Delta \sigma_{2}^{-1}=\sigma_{1}^{-1} \Delta .
\end{array}
$$

Given a braid $B$, let $B^{\prime}$ be the braid obtained by switching all 1 indices with 2 indices; for example if $B=\sigma_{1}^{3} \sigma_{2}^{-1} \sigma_{1}$, then $B^{\prime}=\sigma_{2}^{3} \sigma_{1}^{-1} \sigma_{2}$. So we have

$$
\Delta B=B^{\prime} \Delta
$$

and

$$
\Delta B \Delta B=\Delta^{2} B^{\prime} B
$$

Recall that the topological entropy and the braiding factor can be interpreted as assuming a braid pattern repeats $n$ times; the stretching of a material line in a mixing flow grows exponentially with $n$ (3.6). Thus to understand the behaviour of the braiding factor, we need to understand what happens when a braid is repeated (see figure 2).

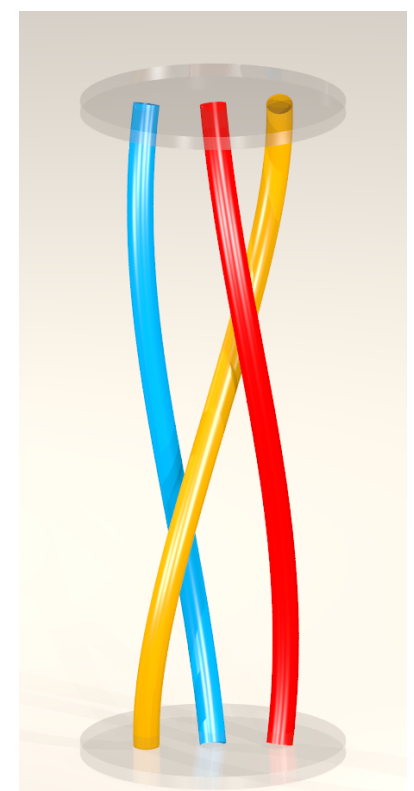

(a)

(b)

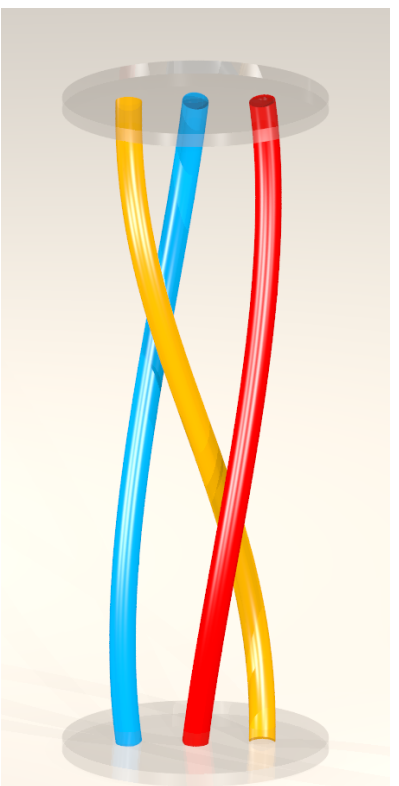

(c)

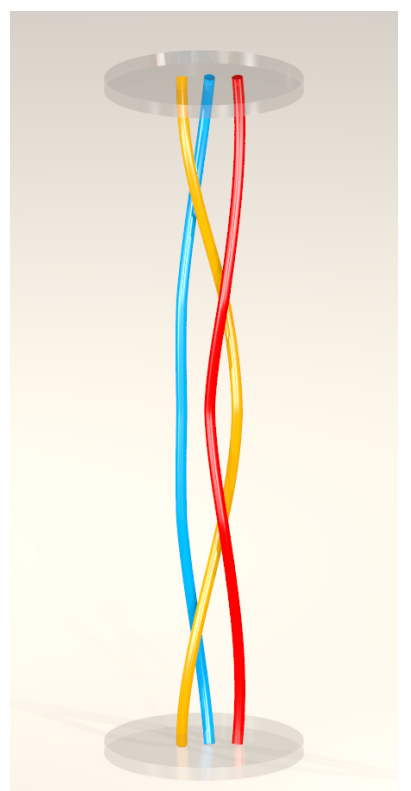

Fig. 2. (colour online) (a) The braid $M=\sigma_{1} \sigma_{2}^{-1}$. (b) The braid $M^{\prime}=\sigma_{2} \sigma_{1}^{-1}$. (c) The braid $M M^{\prime}=\sigma_{1} \sigma_{2}{ }^{-1} \sigma_{2} \sigma_{1}{ }^{-1}=I$. The last braid can be smoothed to three straight lines. 
For example, consider the braid

$$
M=\Delta \sigma_{1}^{a} \sigma_{2}{ }^{-a},
$$

and let it repeat:

$$
\begin{aligned}
M M & =\Delta{\sigma_{1}}^{a} \sigma_{2}{ }^{-a} \Delta \sigma_{1}{ }^{a} \sigma_{2}{ }^{-a}, \\
& =\Delta^{2}{\sigma_{2}}^{a}{\sigma_{1}}^{-a} \sigma_{1}{ }^{a} \sigma_{2}{ }^{-a}, \\
& =\Delta^{2} .
\end{aligned}
$$

By repeating this braid we note that we have lost the entire braid other than the $\Delta^{2}$ term. This vanishing braid occurs whenever our initial braid has an odd power of $\Delta$. However if the power of $\Delta$ is even, there would be no cancellation of the braid.

Thus in any problem where a braid can acquire net rotations, such as in our rotating fluid problem, there is expected to be some erratic behaviour in the braiding factor as the power of $\Delta$ in $M^{(P)}$ changes from odd to even. the origin of this behaviour can be seen in figure 5 (a). Thus we modify the braiding factor by removing the dependence on powers of $\Delta$ altogether. We achieve this by redefining the braiding factor as follows:

$$
\lambda(t)=\max \left(\left\{\mid \text { eigenvalues of } M^{(P)}|,| \text { eigenvalues of } \Delta M^{(P)} \mid\right\}\right) .
$$

The result significantly smooths the plot of $\lambda(t)$ vs. time, as seen in the next section.

\section{Steady kinematic flow simulations}

In this section we examine the braiding properties of the inviscid kinematic flow field generated by the vorticity field (1.1) with $R=\infty$ in each external strain field $\psi_{\text {ext1 }}$ and $\psi_{\text {ext2}}$. These steady results will provide an example flow field where we can robustly examine the braiding diagnostic using particles within the flow and link the regions of rapid braiding to regions of chaos within the flow. Here we set the Reynolds number $R=10^{10}$, so that in the time frame of the simulations, the vortex (1.1) does not spread, so we are able to integrate (2.4) for a sufficient amount of time to see interesting behaviour in the vortex.

As the vortex does not spread in space, we can produce Poincaré sections of the velocity field to show how individual particles placed in the flow move as the strain field is applied. These plots for the flow $\psi_{1}=\psi_{0}+\psi_{\text {ext1 }}$ can be seen in figure 3 and for the flow $\psi_{2}=\psi_{0}+\psi_{\text {ext2 }}$ in figure 4 , both for (a) $A=0.01$, (b) $A=0.02$ and (c) $A=0.03$. These figures are produced by integrating the 
equations (2.4) for 20 evenly spaced particles placed along the $y$-axis, over 2000 periods of the strain field and plotting the position of the particle at the end of each period. In figure 3 we see that the strain field $\psi_{\text {ext1 }}$ creates cat's eyes in the vortex which are clearly visible at the radius $r_{\text {ext }}$ which satisfies $\alpha\left(r_{\text {ext }}\right)=1\left(r_{\text {ext }}=0.399\right.$ in this case $)$. The cat's eyes increase in size as the

(a)

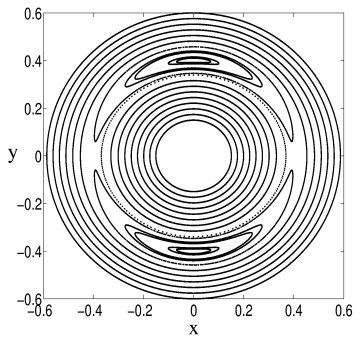

(b)

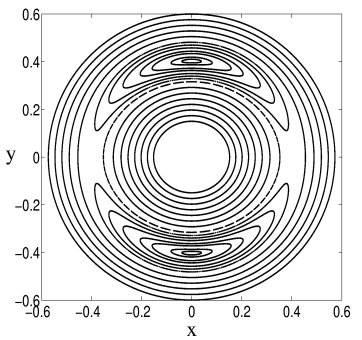

(c)

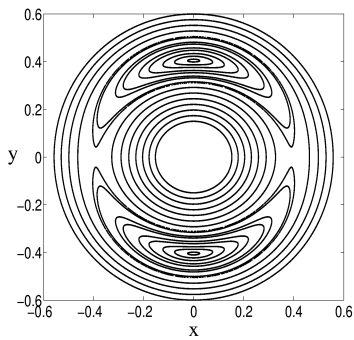

Fig. 3. Poincare sections of the flow field $\psi_{1}=\psi_{0}+\psi_{\text {ext1 }}$ for (a) $A=0.01$, (b) $A=0.02$ and (c) $A=0.03$. The cat's eyes present occur at $r=r_{\text {ext }}$ which satisfies $\alpha\left(r_{\text {ext }}\right)=1$.

amplitude value $A$ increases, but it is clear that these flow fields contain no regions of chaos. Therefore we should expect no exponential increase in the braiding factor as observed in non-chaotic flows by Thiffeault (2005).

(a)

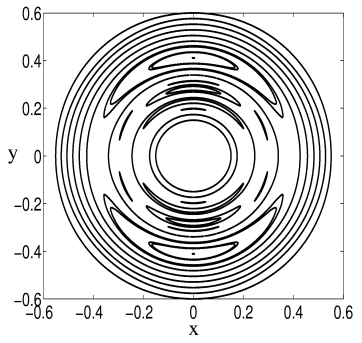

(b)

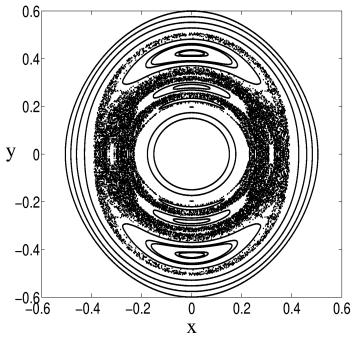

(c)

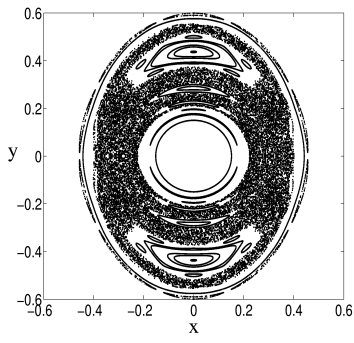

Fig. 4. Poincare sections of the flow field $\psi_{2}=\psi_{0}+\psi_{\text {ext2 }}$ for (a) $A=0.01$, (b) $A=0.02$ and (c) $A=0.03$.

For the multiple cat's eye forcing (2.7), we see that for small amplitude values in figure 4(a) the multiple cat's eyes are clearly visible at many radii, located between $r=0$ and $r=r_{\text {ext }}$. Between the cat's eyes lies a coherent flow field with no visible regions of complex Lagrangian motion. However as we increase the amplitude of the forcing to $A=0.02$ and 0.03 in figures 4 (b) and $4(\mathrm{c})$ respectively, the cat's eyes overlap with one another and give regions of chaos within the vortex. This resonance overlap phenomenon (Lichtenberg and Lieberman, 1992) is a common way to generate chaos in simple systems and the resulting flow will produce regions of braiding with a braiding factor which grows exponentially in time. This type of external forcing which produces multiple cat's eyes within a fluid flow is related to random forcings which occur naturally in flows such as the atmosphere or turbulence. Random forcings do not contain a discrete selection of forcing frequencies, but rather a continuous spectrum of all frequencies and so the resonance overlap phenomena naturally 
occurs. We study the forcing (2.7) as it produces similar effects to a random forcing but is much simpler to generate and study.

Before we make comparisons between the braiding factors of the two velocity fields $\psi_{1}$ and $\psi_{2}$ we first consider the braiding factor for the strain field $\psi_{\text {ext1 }}$. In figure 5 we plot the trio of particles $(3,15,37)$ (defined at the end of $\S 3.2$ ) with $A=0.01$. This strain field only generates one pair of cat's eyes in the

(a)

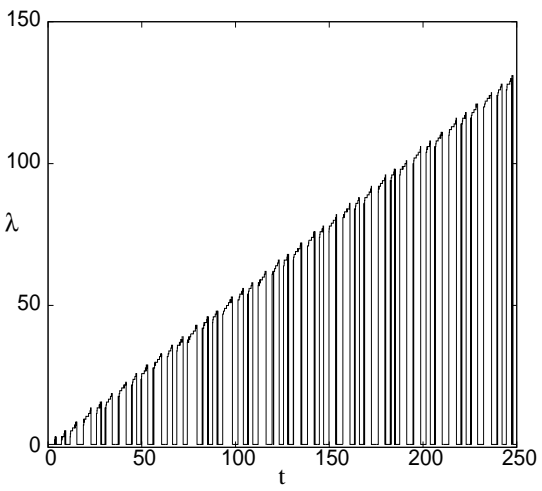

(b)

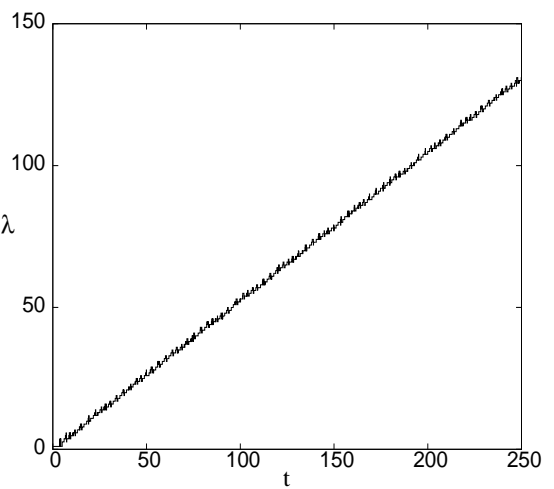

Fig. 5. Plot of the braiding factor $\lambda(t)$ for the trio $(3,15,37)$ in the flow field $\psi_{1}$. In panel (a) $\lambda(t)$ is calculated using method (3.5) and in panel (b) $\lambda(t)$ is calculated using (3.13).

vortex with no chaos in the flow field. In figure 5(a) we plot the braiding factor as given in (3.5) which is the largest eigenvalue of the matrix $M^{(P)}$, and in this case $\lambda(t)$ grows linearly in time and regularly returns to unity as the vortex rotates. This behaviour is almost identical to that seen by Thiffeault (2005) (cf figure 2(a) of his study).

However, this rapid returning to unity is due to the rotation of the vortex, and can be removed by taking into consideration the effects of rotation on Burau matrices, as discussed in the previous section. Figure 5(b) plots the modified braiding factor for the trio in figure 5(a) using the definition (3.13). This braiding factor contains all the same important information as the first braiding factor, but does not keep returning to unity. The graph becomes smoother and it is now possible to plot more than one braiding factor on the same graph without the figure becoming congested. For these reasons we adopt this modified braiding factor as our definition for the remainder of this paper.

In figure $6(\mathrm{a})$ we plot the braiding factor $\lambda(t)$ for the flow field $\psi_{1}$ with (a) $A=0.01$ for the trios $1-(20,21,22), 2-(10,20,30)$ and $3-(3,15,37)$. These three trios were chosen because result 1 gives three particles very close together and so they will all lie within the chaotic region for the forcing $\psi_{\text {ext2}}$, result 2 gives three particles which are well spread within the vortex and result 3 has three particles which give one of the highest values of $\lambda(t)$ at the final time $t=250$ for the strain field $\psi_{\text {ext } 1}$. These three sets of trios give a good range 
(a)

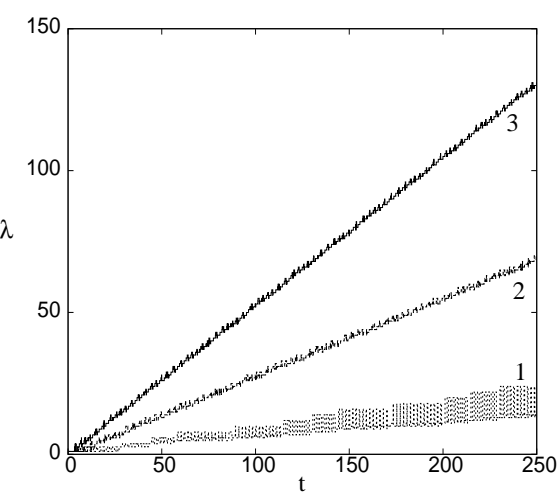

(b)

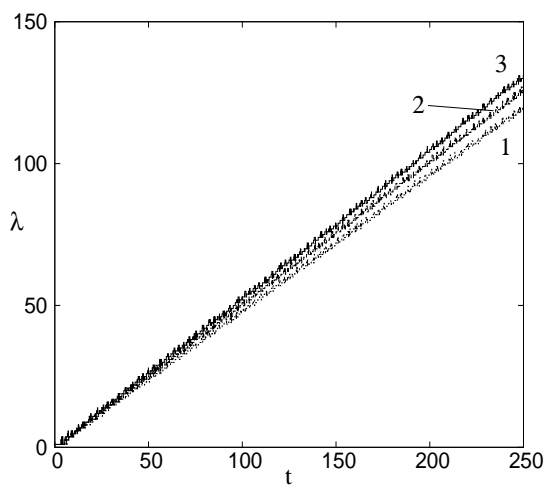

Fig. 6. Plot of the braiding factor $\lambda(t)$ for the flow field $\psi_{1}$ and (a) $A=0.01$ with trios $1-(20,21,22), 2-(10,20,30)$ and $3-(3,15,37)$ and (b) the trio $(3,15,37)$ with $A=0.03, A=0.02$ and $A=0.01$ numbered from $1-3$ respectively.

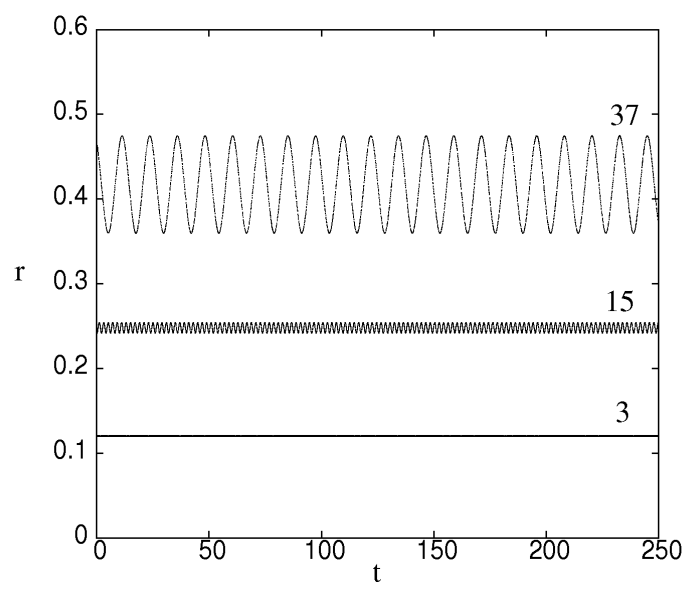

Fig. 7. Plot of $r(t)$ for the three particles from the $(3,15,37)$ trio for $A=0.02$ and $\psi_{1}$.

of behaviours for trios seen in the vortex. We see that results 2 and 3 give a $\lambda(t)$ which grow linearly in time with very little fluctuation, while the result for trio 1 also gives a linear growth but at a much slower rate and with a lot more fluctuation in $\lambda$. This linear growth in $\lambda(t)$ is caused because each particle from the trio is performing a periodic orbit in the vortex. This can be seen in figure 7 which plots the radial coordinates, $r(t)$ from $(2.4)$, of the $(3,15,37)$ trio for $A=0.02$. The $\theta(t)$ plot, also from $(2.4)$, would show periodic behaviour, but the figure is much more congested, so we do not show it here. Figure $6(\mathrm{~b})$ shows the trio $(3,15,37)$ with $A=0.03, A=0.02$ and $A=0.01$ numbered $1-3$ respectively. This panel shows that as the size of the cat's eyes in the flow $\psi_{1}$ increases, the growth rate of the braiding factor actually decreases, while all the time remaining linear, although the overall change in the growth rate is small. This is because even though the cat's eyes increase in size, the flow still remains non-chaotic, therefore we only expect the fluid particles to interchange position along the reference line due to the vortex rotations, and as this is the case for a vortex with smaller cat's eyes too, we 
expect the amount of braiding to be similar in both cases. However, for the forcing (2.7), we expect to see significantly different results when the forcing amplitude $A$ is varied and when we consider different sets of trios.

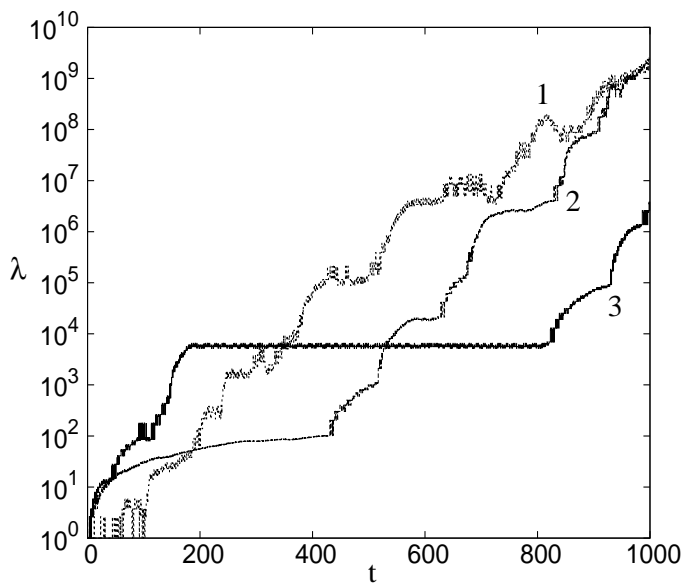

Fig. 8. Plot of the braiding factor $\lambda(t)$ for the flow field $\psi_{2}$ with $A=0.02$ and the trios $1-(20,21,22), 2-(10,20,30)$ and $3-(3,15,37)$.

(a)

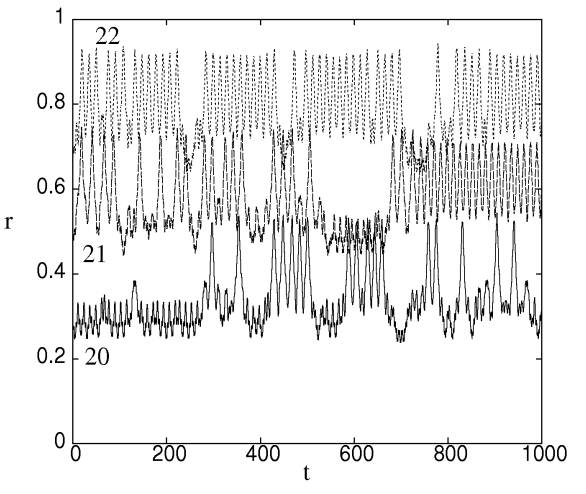

(b)

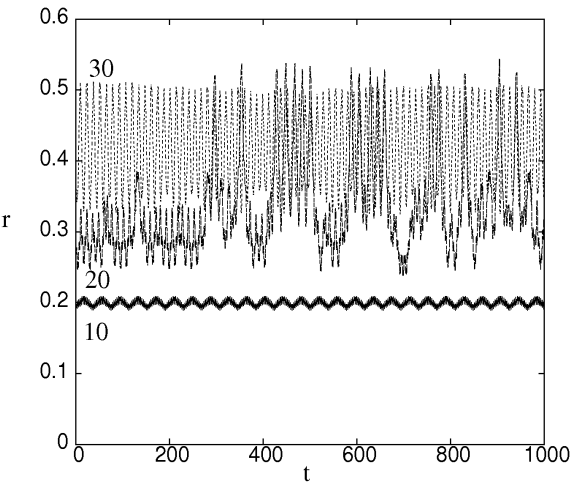

(c)

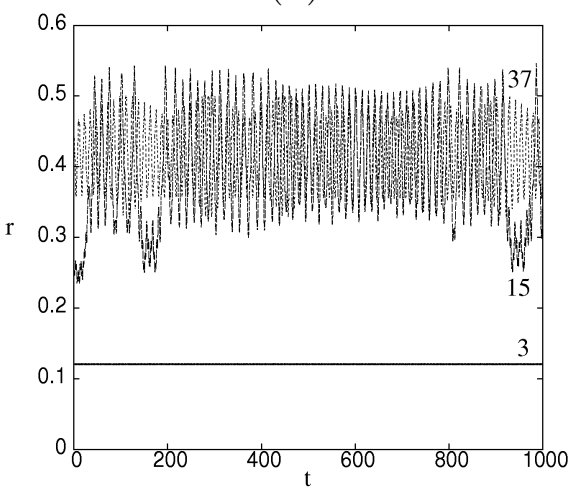

Fig. 9. Plot of $r(t)$ for the flow field $\psi_{2}$ with $A=0.02$ for the trios (a) $(20,21,22)$, (b) $(10,20,30)$ and (c) $(3,15,37)$. In panel (a) the paths 21 and 22 have had the constants 0.2 and 0.4 added to each $r$ point respectively to separate them from one other.

Figure 8 plots the braiding factor $\lambda(t)$ for the flow field $\psi_{2}$ with $A=0.02$ and the three trios $(20,21,22),(10,20,30)$ and $(3,15,37)$ labeled 1 to 3 respectively. We observe that the trio $(3,15,37)$ (result 3$)$ which gave the largest braiding 
factor for the $\psi_{1}$ case in figure 6(a) only has the largest braiding factor up to $t \approx 200$. Beyond this time the braiding factor appears to level off on the log scale in this figure. Actually the braiding factor is still growing in this region, only now the growth is linear in time. After $t=800$ the braiding factor again grows more rapidly. The exponential growth observed in figure 8, which means an intense braiding of the fluid particles, was seen in the work of Thiffeault (2005) for his chaotic flow, and so it is no surprize that we also see it here. What is interesting about our result for the braiding factor is that we see a region of exponential growth, i.e. a very long range of braiding, followed by a region of linear growth, i.e. braiding where all three particles trace periodic orbits and then another region of exponential growth. These periodic orbits can be clearly seen in figure 9(c) which plots $r(t)$. This figure shows that particles 3 and 37 have periodic orbits, and particle 15 undergoes chaotic motion initially and beyond $t=800$. This change in motion agrees well with the change in growth of the braiding factor result in figure 8. Thus, this study shows that we only require one of the particles to undergo chaotic motion to give an exponentially growing braiding factor (positive braiding exponent), while the other two remain in periodic orbits.

The trio $(10,20,30)$ (result 2 ) in figure 8 has a region of no braiding from $t=0$ to $t \approx 400$ where we see a region of linear growth for $\lambda(t)$, then we observe a region of rapid exponential growth for $\lambda$ as particle 20 moves into a chaotic orbit to produce a large amount of braiding in the vortex which can be seen in figure 9 (b). The result 1 , which is for three particles very close to one another, has an exponential $\lambda(t)$ initially and this function remains exponential over the time interval considered. This exponential growth of $\lambda(t)$ is due to all three particles producing chaotic orbits, so if one of the particles falls into a periodic orbit, then there is a second particle still tracing a chaotic orbit to give the positive braiding exponent. One thing to notice for these results is that all of them contain regions of linear growth of $\lambda(t)$ (i.e. poor mixing) some of which are long (results 2 and 3) and some of which are much shorter as in result 1. This sort of behaviour is to be expected of a flow which has regions of chaos and regions of no chaos, as the exponential growth of $\lambda(t)$ only requires 1 particle of the trio to have a chaotic orbit. Therefore, if this particle then becomes close to the edge of the chaotic region, it can spend some time close to a periodic orbit and so the braiding rate drops until the particle makes its way back into the main part of the chaotic region where the braiding rate, $\lambda(t)$, then grows exponentially again. This is one of the major difficulties with trying to measure the amount of mixing in these types of flows. Some of these problems can be overcome by considering the 50-braid braiding factor (see later) but even these results are not as clean as one would like.

In figure 10 we plot the trios $(10,20,30)$ and $(3,15,37)$ in panels $(\mathrm{a})$ and $(\mathrm{b})$ respectively for $A=0.01,0.02$ and 0.03 numbered 1 to 3 in each panel. For each trio the $A=0.01$ result gives a linear growth in $\lambda(t)$ but this could have 

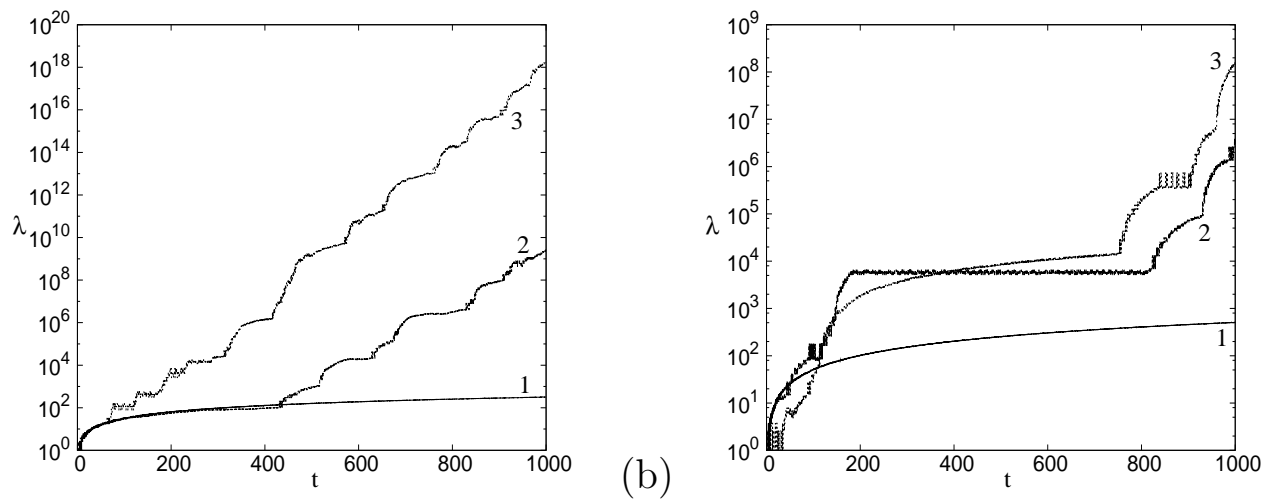

Fig. 10. Plot of the braiding factor $\lambda(t)$ for the flow field $\psi_{2}$ and (a) the trio $(10,20,30)$ and (b) the trio $(3,15,37)$ for $1-A=0.01,2-A=0.02$ and $3-A=0.03$.

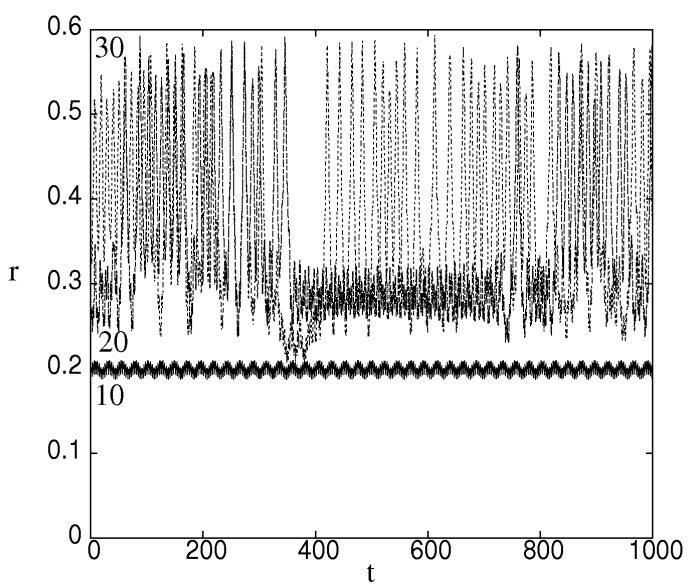

Fig. 11. Plot of $r(t)$ for the flow field $\psi_{2}$ with $A=0.03$ and the trio $(10,20,30)$.

been anticipated from the Poincaré section in figure 4(a) in which the vortex contains no visible regions of chaos. For the trio $(10,20,30)$ in panel (a) we see that as the forcing amplitude increases, the braiding factor again grows linearly at first and then once particles 20 and 30 move into a region of chaos, the braiding factor grows exponentially in time, interspersed with regions of linear growth (See figure 11 for a plot of $r(t)$ for this trio of particles where it is difficult to distinguish between particles 20 and 30 as they trace out their respective paths). This exponential growth of $\lambda(t)$ occurs earlier as $A$ increases but the overall braiding exponent remains similar to the $A=0.02$ result. The exponential growth happens earlier because, as can be seen in figure 4 , the region of chaos increases in size as $A$ increases, so particles are subject to chaotic orbits more readily. In figure 10 (b) the result for $A=0.02$ (result 2) has chaotic mixing, and an exponential growth in $\lambda(t)$ for $t<200$ and then a linear growth and no mixing until $t \approx 800$ where it again grows exponentially in time. One might expect as $A$ is increased to 0.03 that there is more mixing within the flow, but in fact for this trio the exponential growth in $\lambda(t)$ is very similar for both $A=0.02$ and 0.03 . However, this could be due to the random initialization of $\theta_{i}$ placing the particles in different positions on the 
same radius.

(a)

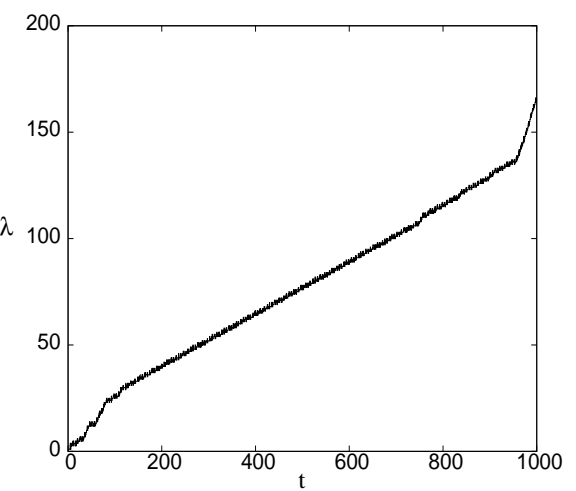

(b)

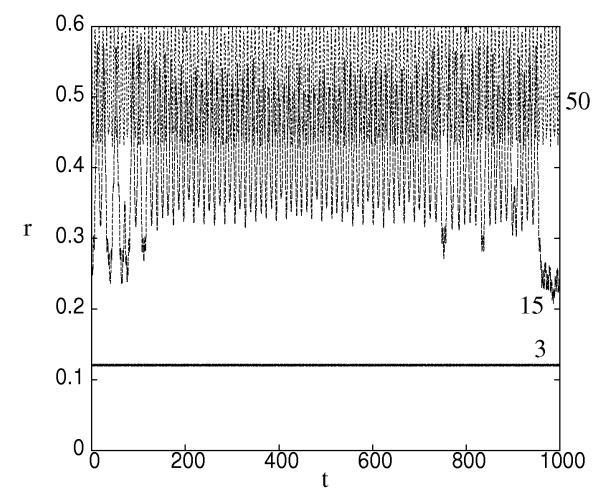

Fig. 12. Plot of the braiding factor $\lambda(t)$ for the flow field $\psi_{2}$ with $A=0.03$ and the trio $(3,15,50)$ and (b) the corresponding $r(t)$ for this trio.

One important thing to note when analysing the 3-braid braiding factor of a flow is, we have to be very careful about which trio of particles we pick. In figure $12(\mathrm{a})$ we pick the trio $(3,15,50)$ to analyse the chaotic forcing $(2.7)$ for $A=0.03$ and we find that the braiding factor grows linearly with time, but we know from the Poincaré section in figure 3(c) that this flow contains a large region of chaos, so one might expect an exponentially growing braiding factor. However, this case gives an example of a 3 -braid where all three particles are performing approximate periodic orbits as seen in the $r(t)$ plot in figure 12(b). Therefore, if we try to infer information about the flow using just these three particles then we might incorrectly assume that there is no chaos in the flow. For this example we know that this is incorrect because we have the Poincaré section in figure 3(c) to show us otherwise, but if one does not have any information about the flow field, such as the Labrador sea example in Thiffeault (2010), then one could infer incorrect characteristics of the flow if the wrong trio is analysed. The conclusion here is, the 3-braid gives an excellent indication of how much mixing is going on in specific regions of the flow, but to get an overall impression of the mixing properties of the flow, one should consider the motion of more particles and thus consider a larger $p$-braid braiding factor.

In figure 13(a) we plot the $p$-braid braiding factor for the flow field $\psi_{2}$ with $A=0.02$ and $p=5,10,25$ and 50 numbered $1-4$ respectively. Here rather than pick specific particles, we choose equally spaced particles from (3.1). This gives us a braiding factor for the part of the flow we are considering i.e. $0.1 \lesssim r \lesssim 0.6$. Ideally we would have particles in the entire flow field, but as all the interesting behaviour is in this region we don't expect the braiding factor to change much with the inclusion of particles outside this region. As we increase $p$, we see that the braiding exponent increases and as $p$ becomes large the braiding exponent tends to the same value for all $p$, but with a slow increasing drift. This result is in accord with the findings of Thiffeault (2005) who also found this slow drift in the braiding exponent for large $p$. In panel (a) 


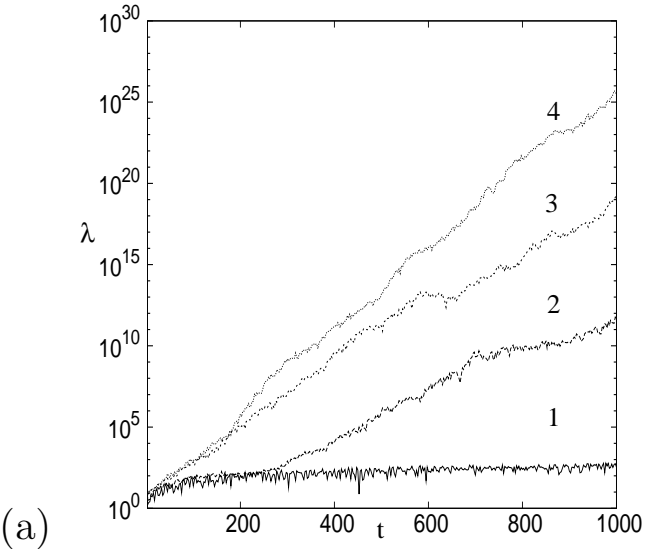

(a)

Fig. 13. Plot of (a) the $p$-braid braiding factor for $\psi_{2}$ with $A=0.02$ for $p=5,10,25$ and 50 numbered $1-4$ respectively. Panel (b) shows the 50 -braid braiding factor before it has been artificially smoothed to give result 4 in panel (a).

all the braiding factors have been artificially smoothed by considering a 1000 point average of the original data which has a time spacing of $\Delta t=2.5 \times 10^{-3}$, so as to remove the rapid oscillations seen in panel (b) which shows the $p=50$ braiding factor which has not been smoothed. The rapid oscillations are due to the increased number of crossings compared to the 3 -braid plots, and unlike the 3-braid, we cannot easily remove some of these oscillations using an argument similar to that in $§ 3.3$. This is because when we consider more than three particles in the flow, the number of crossings increases greatly, and just removing half turns, as we did for the 3-braid problem, makes no significant difference to the braiding factor. To smooth out the braiding factor one would also have to remove partial turns where only some particles have interchanged positions, so this would involved a more complicated form of (3.13) where the number of elements in the set to be maximised is much larger. This would greatly increase the running time of the code and hence make it less practical.

The use of the $p$-braid with $p$ large becomes obvious if one is interested in calculating the approximate instantaneous gradient of the lines in figure 13(a) to give the time dependent braiding exponent $\Lambda(t)$. However, the purpose of this paper is to examine the robustness of the use of braids in more 'realistic' fluid flow problems. At this stage we are not interested specifically in the value for the braiding exponent, i.e. we don't want to state that a flow with $A=0.02$ has a braiding exponent of 0.4 for example, we just want to know if $\lambda(t)$ is linear or exponential in time, and whether or not this behaviour changes as the flow evolves in time. 


\section{Unsteady kinematic flow and dynamical flow simulations}

The aim of this research is two fold: Firstly to understand the mixing properties of vortices by examining their braiding factors, and secondly to compare the results of the kinematic integral flow model with those of the dynamical flow model to examine similarities and differences. However as discussed in $\S 1$, we wish to compare the kinematic flow results with the dynamical flow results of Turner and Gilbert (2009) who show that when $r_{\text {ext }} \approx r_{\text {cat }}$ in a viscously spreading vortex, there is a large feedback of the resonant harmonics in the vortex which grow rapidly changing the azimuthally averaged vortex profile and producing visual signs of mixing. The purpose of this study is to quantify whether or not mixing actually exists in this model. The kinematic flow approach will not experience this resonance, and so it is interesting to see the difference between the kinematic and dynamic flow results. Thus in the next section we examine braiding results for a viscously spreading vortex with Reynolds number $R=10^{4}$ and we compare these results with the non-linear dynamical flow results, of Turner and Gilbert (2009). Before we begin making comparisons between the kinematic integral flow model and dynamical flow model results, let us first examine the braiding factor of the kinematic flow model and make comparisons with the steady results in $\S 4$, to highlight the key differences.

\subsection{Kinematic integral flow}

To see what the flow field looks like for the kinematic flow, we plot Poincaré sections at various times in figures 14 and 15. As the vortex is viscously spreading for this value of $R$, we produce Poincare sections of what the flow field would look like if it were steady at the various times (a) $t=100$, (b) $t=200$, (c) $t=300$ and (d) $t=350$ for the velocity field $\psi_{1}$ with $A=0.02$ in figure 14. To produce these plots we take the form of the vortex at the appropriate time from (1.1) and then produce a steady form of the Poincaré section. Thus we are taking snapshots of the flow field at a time $t$.

We observe that as the vortex spreads, the cat's eyes move inward to smaller radial values, until eventually at $t=t_{\mathrm{van}}=382$ they disappear altogether. We note that as these cat's eyes move towards the origin, the forcing still does not generate any complex Lagrangian motion in the flow. However, at a time close to when the cat's eyes vanish, there may be interaction between the cat's eyes themselves.

In figure 15 we plot the same panels as in figure 14, except this time we plot the flow field $\psi_{2}$ with $A=0.02$ which does generate complex Lagrangian motion 
(a)

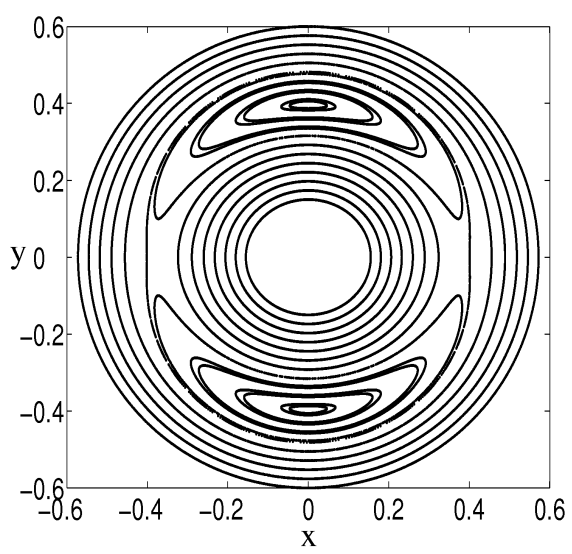

(c)

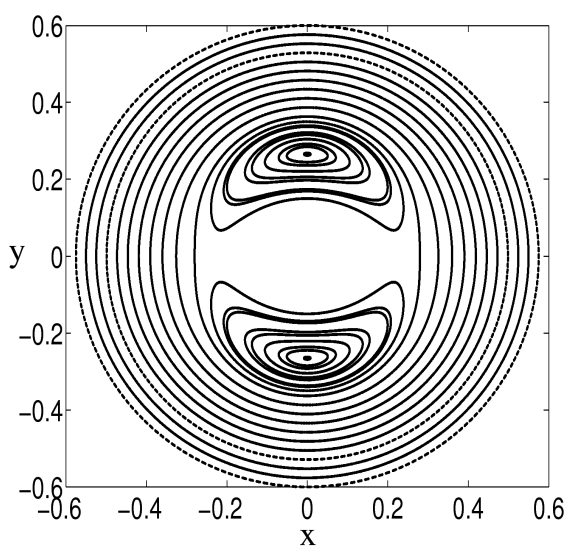

(b)

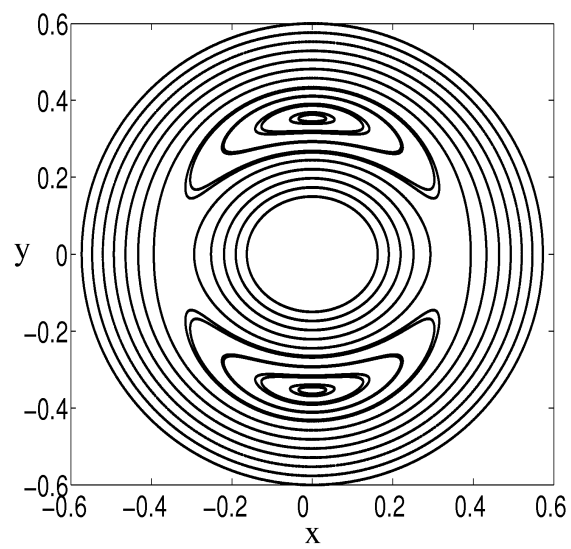

(d)

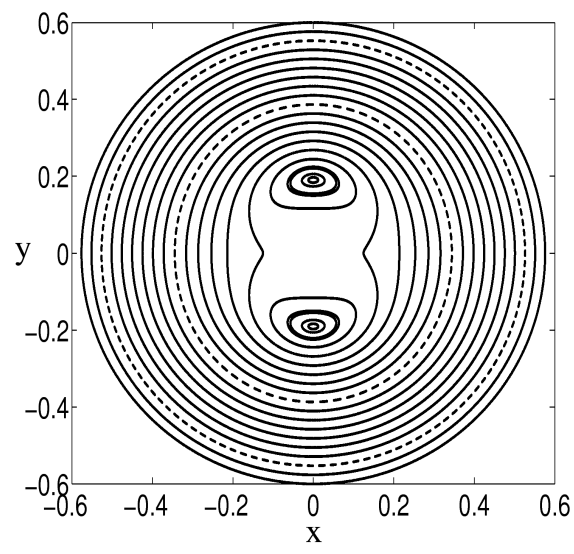

Fig. 14. Poincaré sections for the flow field $\psi_{1}=\psi_{0}+\psi_{\text {ext1 }}$ with $R=10^{4}$ and $A=0.02$ at (a) $t=100$, (b) $t=200$, (c) $t=300$ and (d) $t=350$.

in the vortex. In this case we see that at early times we have complex motion in the vortex, exactly as in the steady case, but by the time $t=200$ in panel (b) we see that the flow has become periodic everywhere and we are left with a vortex with a large set of cat's eyes, as in the $\psi_{\text {ext1 }}$ forcing case in figure 14(b), and a smaller set close to $r=0.2$. The small set of cat's eyes remaining near $r=0.2$ soon disappear and for the remaining times in panels (c) and (d) we have velocity fields which look like their $\psi_{1}$ counterparts of figure 14 . Hence for these flows we expect to see large amounts of braiding at early times, $\lambda(t)$ growing exponentially in time, but less braiding later, linear $\lambda(t)$, once the vortex spreads and the complex motion disappears. At this point we then expect the flow to behave like the $\psi_{1}$ flow.

In figure 16 we plot the 3 -braid braiding factor for $A=0.02$ for the flow fields (a) $\psi_{1}$ and (b) $\psi_{2}$ for three different trios. For the flow field $\psi_{1}$, both the trios $(3,15,37)$ (result 1 ) and $(10,20,30)$ (result 2) have a similar behaviour, in that their respective braiding factors $\lambda(t)$ grow linearly up to $t \approx 200$ and then there is a brief rapid increase in $\lambda(t)$, before this growth slows down and $\lambda(t)$ grows linearly again at later times. The trio $(20,21,22)$ (result 3$)$ does not see the same increase around $t=20$, and instead just grows linearly for all times, 
(a)

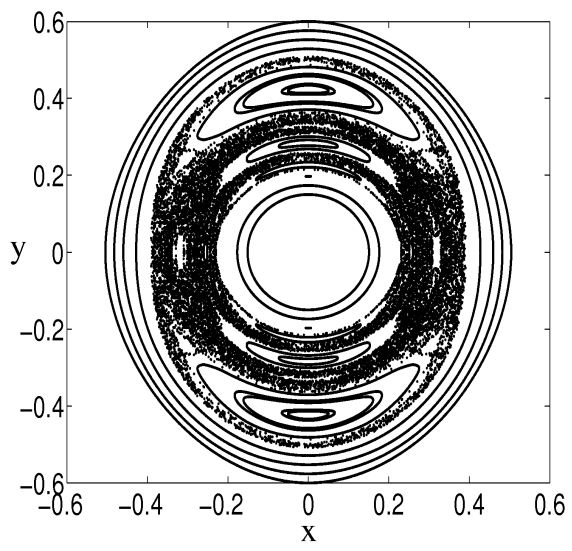

(c)

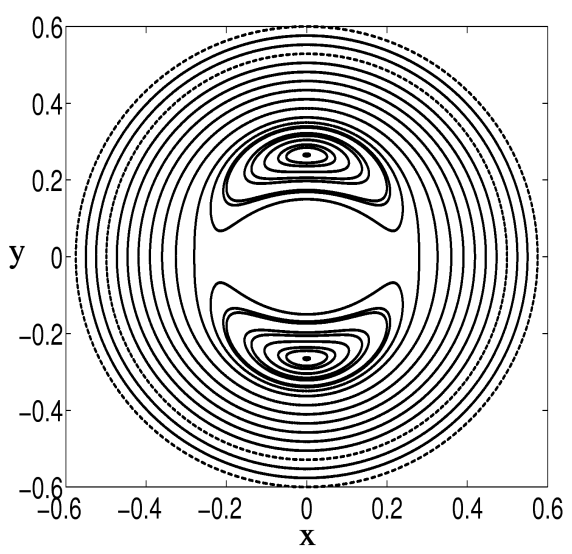

(b)

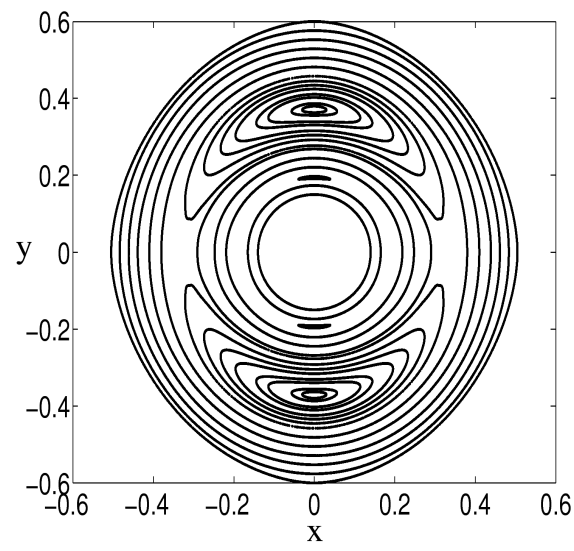

(d)

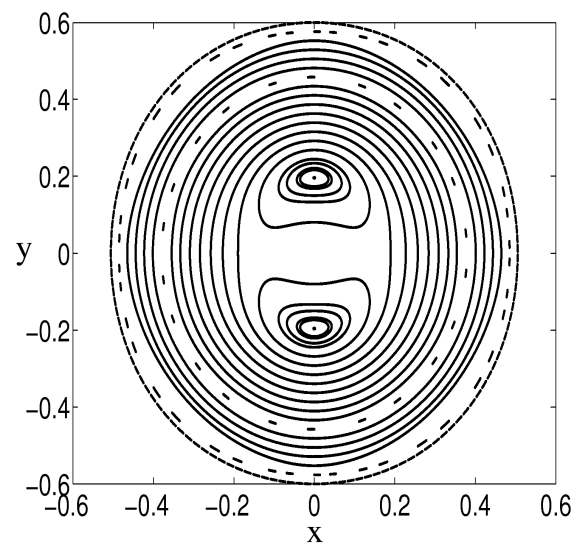

Fig. 15. Poincaré sections for the flow field $\psi_{2}=\psi_{0}+\psi_{\text {ext2 }}$ with $R=10^{4}$ and $A=0.02$ at (a) $t=100$, (b) $t=200$, (c) $t=300$ and (d) $t=350$.

(a)

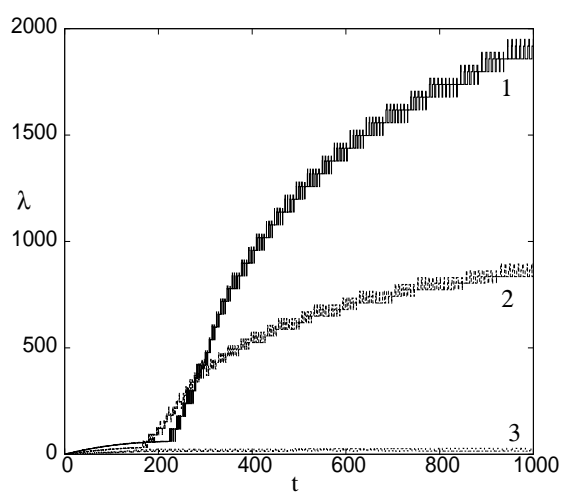

(b)

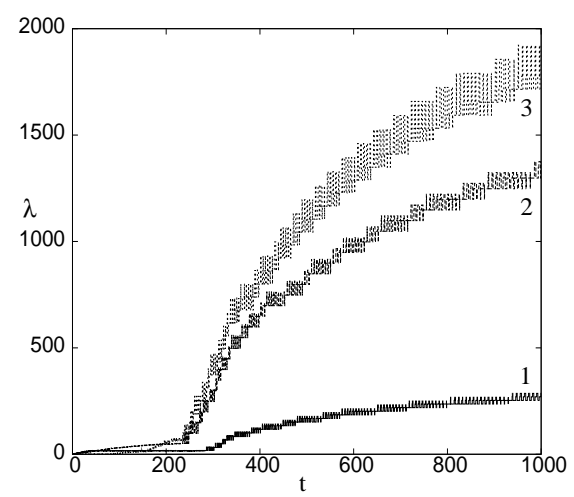

Fig. 16. Plot of the braiding factor $\lambda(t)$ for the case $R=10^{4}$ and $A=0.02$ for the flow fields (a) $\psi_{1}$ and (b) $\psi_{2}$. In each panel the lines represent the trios $1-(3,15,37)$, $2-(10,20,30)$ and $3-(20,21,22)$

just as for the steady case in $\S 4$. The increase in $\lambda(t)$ is due to the particles being shifted around in the vortex as it spreads and appears to correspond to the time when two particles radial values coincide. This can be seen for the $(3,15,37)$ trio in figure $17(\mathrm{a})$ where we plot $r(t)$. We see that the particle numbered 37 lies within a cat's eye initially and so gets drawn towards the 
(a)

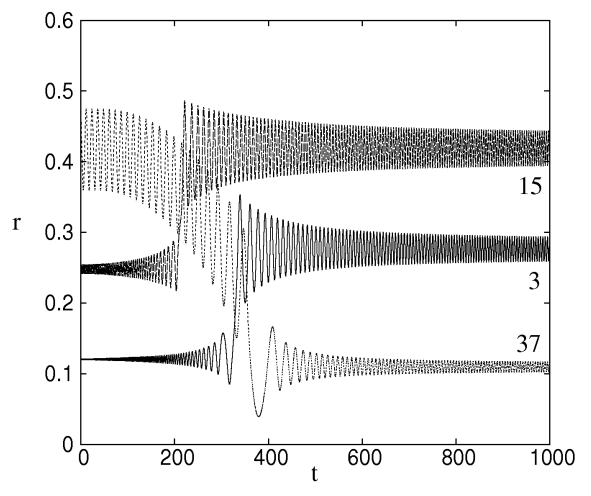

(b)

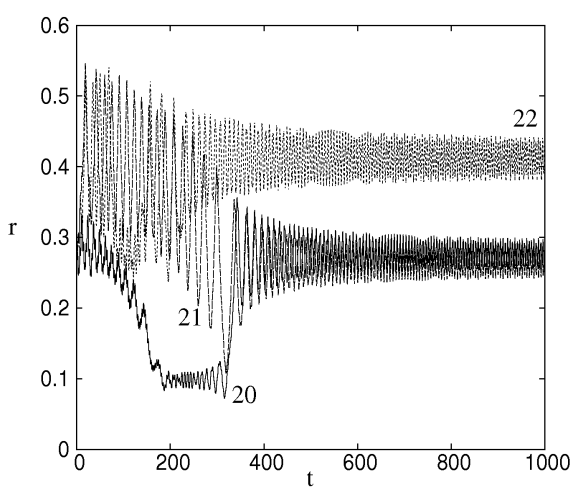

Fig. 17. Plot of $r(t)$ for (a) the trio $(3,15,37)$ in the flow field $\psi_{1}$ with $R=10^{4}$ and (b) the trio $(20,21,22)$ in the flow field $\psi_{2}$ with $R=10^{4}$. The amplitude $A=0.02$ in both panels.

centre of the vortex as time increases, and eventually escapes the cat's eye at $t \approx 382$ when they disappear. The other two particles on the other hand, which lie inside the radius of the cat's eyes $r_{\text {ext }}$, get thrown outwards as they meet the inward moving cat's eyes. However, as there is no complex Lagrangian motion in the strain field, the particles continue to complete periodic orbits as the vortex evolves. Maybe surprisingly, the trios considered here behave very similarly in the flow field $\psi_{2}$ for which $\lambda(t)$ is plotted in figure 16(b). The only obvious difference here is that now a different trio gives the largest value of $\lambda(t)$ at large times. When we see how the radius values of the individual particles move in figure 17(b), we see they move similarly to the $\psi_{1}$ case. The early complex motion moves the particles around in the vortex, and particles 20 and 21 become trapped within different cat's eyes and move inwards, while particle 22 remains at a larger radii. Unlike the steady case, the particles are not given enough time in the 'chaotic' region to braid sufficiently which would give a positive braiding exponent, and instead the particles have periodic orbits. This is why the results are similar to the $\psi_{1}$ flow case.

(a)

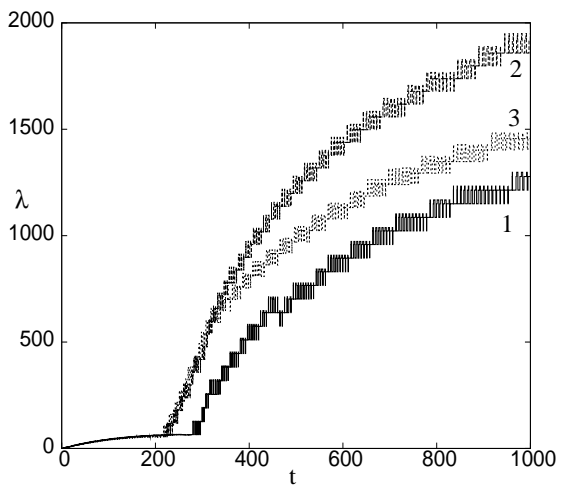

(b)

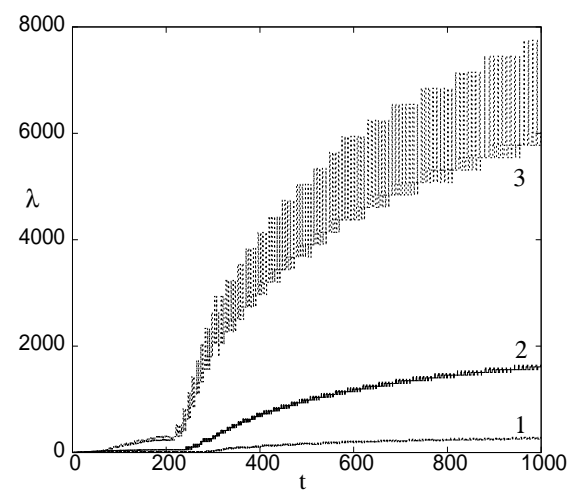

Fig. 18. Plot of the braiding factor $\lambda(t)$ with $R=10^{4}$ for the flow fields (a) $\psi_{1}$ and (b) $\psi_{2}$ for the trio $(3,15,37)$. In each panel the lines represent the forcing amplitudes $A=0.01,0.02$ and 0.03 numbered 1 to 3 respectively.

Now that the cat's eyes are not static in the vortex, and move towards the 
centre of the vortex, this can affect various aspects of the braiding factor evolution. For example in figure 18(b), which shows $\lambda(t)$ for the trio $(3,15,37)$ for the flow $\psi_{2}$ for $A=0.01,0.02$ and 0.03 numbered $1-3$ respectively, we see that as $A$ is increased, then so too does $\lambda(\mathrm{t})$. While for the flow $\psi_{1}$ in figure 18(a) we find that the $A=0.02$ case has a larger value of $\lambda$ than the $A=0.03$ case at large times. This could just be a consequence of the starting position of each particle in the different runs, as we note that the initial $\theta$ position of each particle is random. However, these results highlight the need to consider $p$-braid braiding factors for these flows with $p>3$, so as to get a good quantitative information from the entire vortex.

\subsection{Comparison of the kinematic integral flow model and the dynamical flow model}

We now have a large amount of information about how braiding can be used to determine mixing properties of flows. In this section we use this information to make a comparison between the kinematic integral flow model in $\S 5.1$ and the dynamical flow simulations of Turner and Gilbert (2009).

The fully nonlinear simulations in Turner and Gilbert (2009) solve the nondimensional Navier-Stokes equations in cylindrical polar coordinates

$$
\begin{gathered}
\partial_{t} \omega+J\left(\omega, \psi+\psi_{\text {ext }}\right)=R^{-1} \nabla^{2} \omega, \quad \nabla^{2} \psi=-\omega \\
r J(a, b)=\left(\partial_{r} a\right)\left(\partial_{\theta} b\right)-\left(\partial_{\theta} a\right)\left(\partial_{r} b\right), \quad \psi_{\text {ext }}=q(t) r^{m} e^{i m \theta}+\text { c.c }
\end{gathered}
$$

by time stepping (5.1) and seeking solution for $\omega(r, \theta, t)$ and $\psi(r, \theta, t)$ in the form of a truncated sum of Fourier harmonics

$$
\omega(r, \theta, t)=\sum_{k=-Q}^{Q} \omega_{m k}(r, t) e^{i m k \theta}, \quad \psi(r, \theta, t)=\sum_{k=-Q}^{Q} \hat{\psi}_{m k}(r, t) e^{i m k \theta} .
$$

The external strain field $\psi_{\text {ext }}$ in (5.2) with $m=2$ drives the $m=2$ mode of the vorticity field which then excites higher Fourier harmonics, as well as modifying the basic profile $\omega_{0}$ via the nonlinear terms $J\left(\omega, \psi+\psi_{\text {ext }}\right)$ of (5.1). To integrate (5.1) we use the Crank-Nicolson method on the linear terms and the second order Adams-Bashforth method on the nonlinear terms. The discretisation in the radial direction uses a finite difference method with $S$ grid points in the range $0 \leq r \leq r_{0}$, and the Poisson equation for $\psi(r, \theta, t)$ in (5.1) is solved by inverting a tridiagonal matrix system. The code uses $Q$ Fourier harmonics and for this study typical resolution values are $\left(Q, S, r_{0}\right)=$ $(128,1500,6)$ for $R=10^{4}$.

Turner and Gilbert (2009) only consider the effect of the strain field $\psi_{\text {ext1 }}$, and they find that when $r_{\text {ext }} \approx r_{\text {cat }}$, the natural quasi-mode radius in the 
vortex at which cat's eyes can persists with no external forcing, there is a resonance effect in the vortex and the vorticity spreads out rapidly until the cat's eyes vanish. For more information on quasi-modes and the resonant effect the reader is referred to this paper. Although this paper did not consider the external forcing $\psi_{\text {ext2 }}$, we expect similar results to the $\psi_{\text {ext1 }}$ forcing because we saw in figure 15 that the initial complex Lagrangian motion disappears as the vortex spreads leaving a single set of cat's eyes as in $\psi_{\text {ext1 }}$.

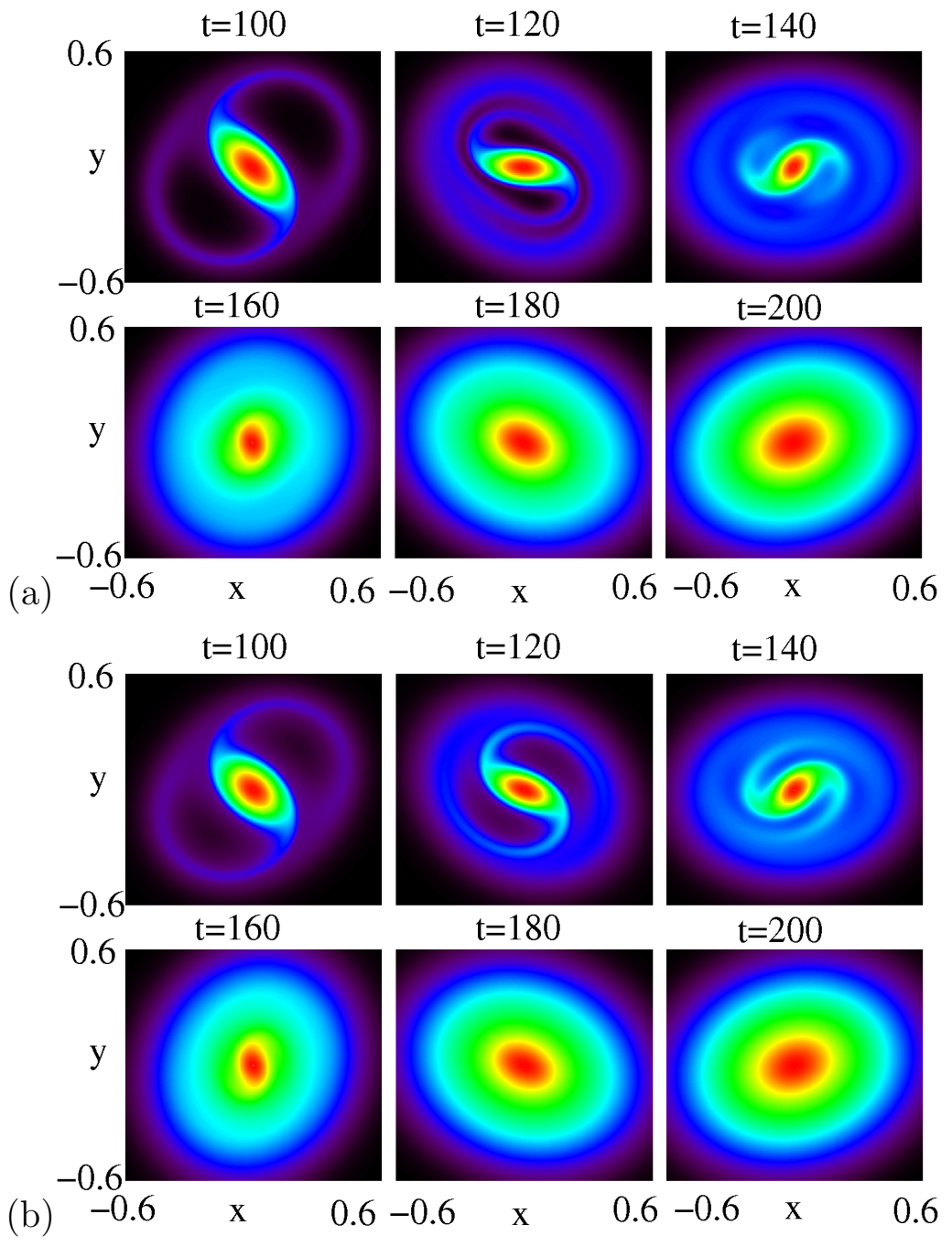

Fig. 19. (colour online) Plot of the vorticity field for the dynamical flow simulations with $R=10^{4}$ and $A=0.03$ for (a) $\psi_{\text {ext1 }}$ and (b) $\psi_{\text {ext2 }}$. In each panel the vorticity has been scaled so that red (dark grey at the origin) denotes the maximum at that time value.

In figure 19 we plot the vorticity field for the nonlinear simulations with $R=$ $10^{4}$ and $A=0.03$ for (a) $\psi_{\text {ext1 }}$ and (b) $\psi_{\text {ext2 }}$. The addition of the complex motion in $\psi_{\text {ext2 }}$ makes very little difference in these figures, possibly because at this Reynolds number the viscous spreading of the vortex is too fast for the complex motion to have a significant effect. However, the complex motion in $\psi_{\text {ext2 }}$ does slow the rate of decrease of $\omega(r=0, t)$ which can be seen in 
figure 20. In figure 20 line 1 gives the slow viscous decrease of the vorticity

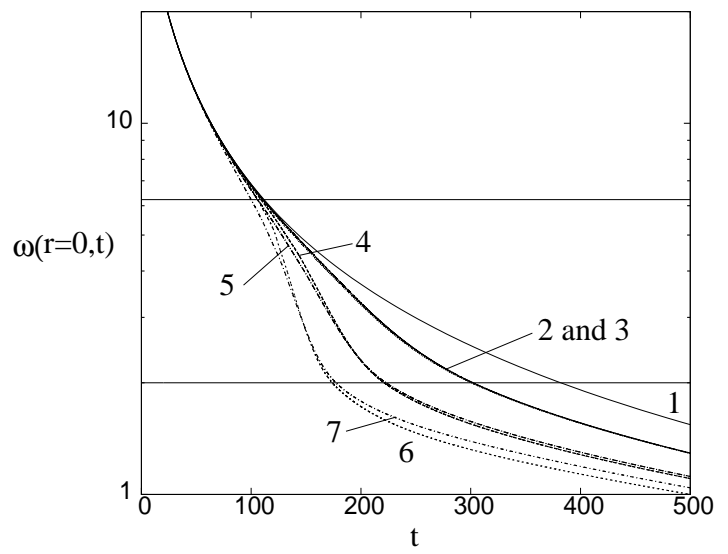

Fig. 20. Plot of the vorticity value at the origin, $\omega(r=0, t)$ for the dynamical flow simulations with $R=10^{4}$. Result 1 is the value taken by the kinematic flow model, results 2 and 3 are for $\psi_{\text {ext1 }}$ and $\psi_{\text {ext2 }}$ with $A=0.01$, results 4 and 5 are for $\psi_{\text {ext1 }}$ and $\psi_{\text {ext2 }}$ with $A=0.02$ and results 6 and 7 are for $\psi_{\text {ext1 }}$ and $\psi_{\text {ext2 }}$ with $A=0.03$. The top horizontal line indicates when $r_{\text {ext }} \approx r_{\text {cat }}$ while the lower horizontal line is when the cat's eyes vanish from the vortex.

at the origin for the kinematic flow model. We can see that the inclusion of the feedback between the external forcing and flow field causes the value of $\omega(r=0, t)$ to drop more rapidly due to the resonance effect discussed earlier. Result 6 which is for $A=0.03$ and $\psi_{\text {ext1 }}$ shows the effect of one set of cat's eyes in the vorticity field as reported in Turner and Gilbert (2009). The new result here is the result 7 which gives $\omega(r=0, t)$ for $\psi_{\text {ext2 }}$, and we see that the inclusion of the complex Lagrangian motion has made the result differ from result 1 earlier, but also that the rapid decline in $\omega(r=0, t)$ is now slower. We try to quantify this difference in behaviour using the 50-braid braiding factor below.

When we look at the 50-braid braiding factor for the external forcing $\psi_{\text {ext1 }}$ in figure 21, we see that both the figures for (a) the kinematic flow simulations and (b) the dynamical flow simulations look very similar. While curves 2 and 3 in each panel seem to increase slightly around the time that the cat's eyes vanish (300 $\leq t \leq 400$ in panel (a) and $100 \leq t \leq 200$ for panel (b)) the growth in $\lambda(t)$ is too rapid to tell whether or not it is exponential in time at this point, giving a positive braiding exponent, which would then imply complex Lagrangian motion in the vortex. The reason we don't see any clear exponential growth could be one of three reasons: firstly there may not actually be any complex motion in the vortex as the cat's eyes vanish, secondly the amount of complex motion might be small, so the rapid spreading of the vortex might not give us enough time to see such an event or thirdly, we did not place particles close enough to the centre of the vortex to see any complex motion. One could increase the Reynolds number in an attempt to see any complex 
(a)

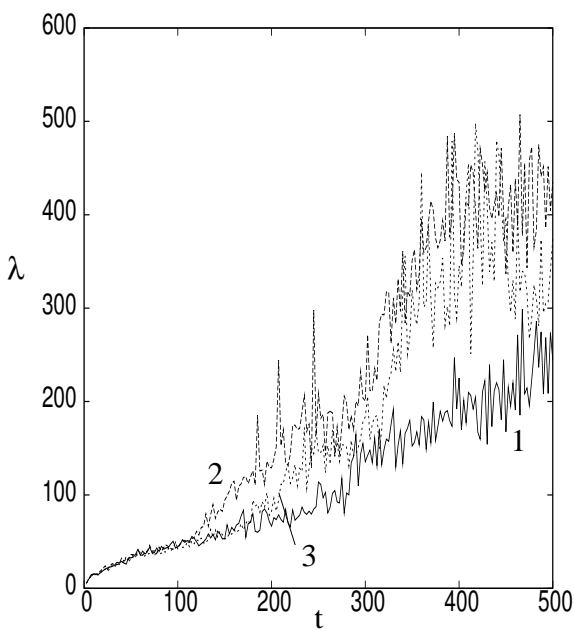

(b)

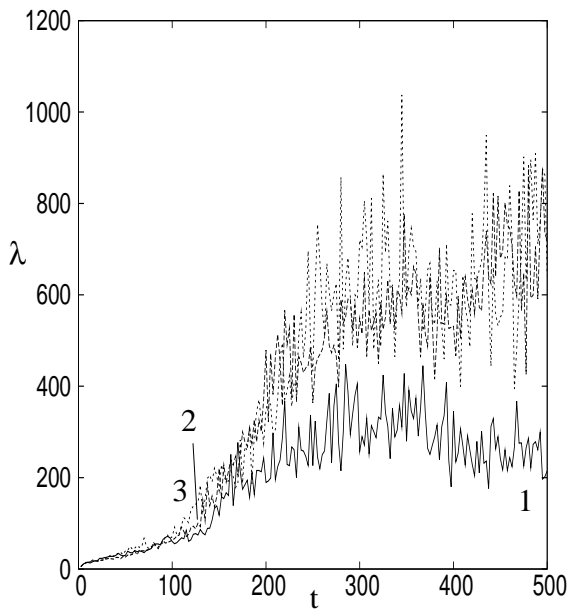

Fig. 21. Plot of the 50-braid braiding factor for the external forcing $\psi_{\text {ext1 }}$ for (a) the kinematic flow simulations and (b) the dynamical flow simulations for $A=0.01,0.02$ and 0.03 numbered $1-3$ respectively.

motion as the cat's eyes vanish, but Turner and Gilbert (2009) showed that even for $R=10^{5}$ (the top end of the computational time for their code) the time spent with the vortex having cat's eyes close to vanishing is very short. An examination of the 50-braid braiding factor for $\psi_{\text {ext2 }}$ in figure 22 shows

(a)

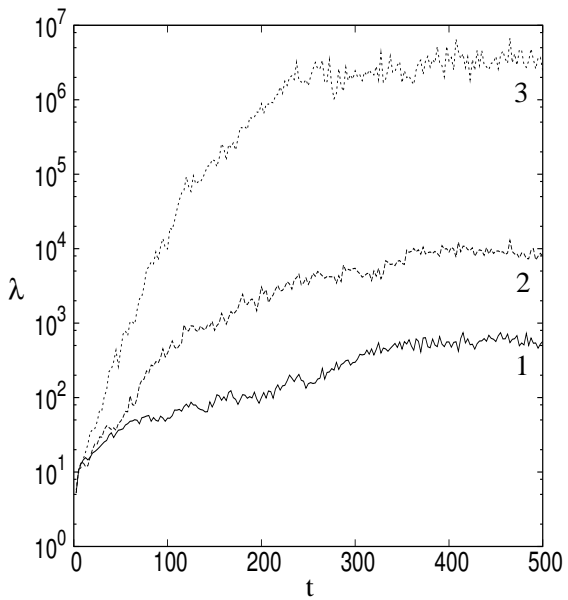

(b)

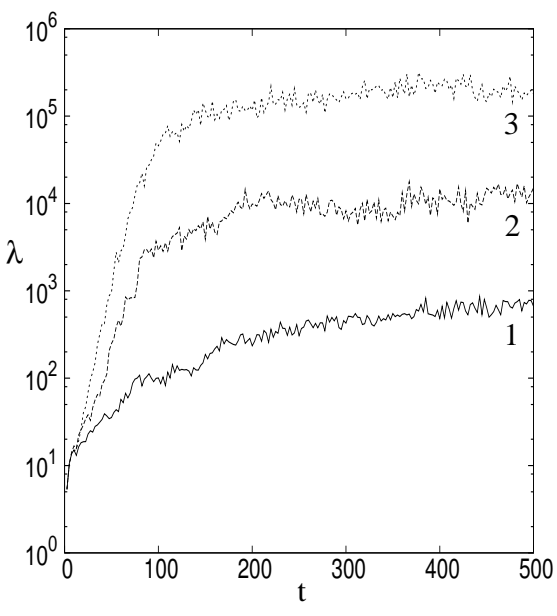

Fig. 22. Plot of the 50-braid braiding factor for the external forcing $\psi_{\text {ext2 }}$ for (a) the kinematic flow simulations and (b) the dynamical flow simulations for $A=0.01,0.02$ and 0.03 numbered $1-3$ respectively.

that this gives a much more interesting result. We find that for the kinematic flow simulations in panel (a) only $\lambda(t)$ for the $A=0.03$ result (result 3) has an obvious region of exponential growth, and this occurs up to $t \approx 200$. Beyond this time the complex motion disappears and so the growth in $\lambda(t)$ becomes linear in time again. The $A=0.02$ result (result 2) has a small region of exponential growth up to $t \approx 100$, but the small amplitude result (result 1) appears to just have linear growth. While the $A=0.01$ result for the dynamical flow simulations in panel (b) appears to grow linearly like its 
kinematic counterpart, the other two results both have regions of exponential growth up to $t \approx 100$ which is the time when the rapid spread of vorticity occurs. This figure also shows that the rate of increase of $\lambda(t)$ with time is greater for the dynamical simulation and, at least for $A=0.03$, the value of $\lambda(500)$ is less than the kinematic value. This means that the material lines in the chaotic region will be stretched more in the kinematic model but the rate of stretching is faster in the dynamic model. The reason they are stretched more in the kinematic model is solely due to the fact that they are stretched for a longer period of time. Approximate values for the braiding exponents of both the $A=0.03$ results are 0.08 and 0.1 for the kinematic integral flow model and dynamical flow model respectively. Therefore the outcome is that the nonlinear feedback of the strain field on the vorticity field suppresses the total amount of braiding (and hence mixing), but the maximum rate at which braiding occurs is increased.

\section{Conclusions and Discussion}

This work studied the effectiveness of using the braiding diagnostic to quantify the amount of mixing within coherent vortical structures. We used the method detailed in Thiffeault (2005) which represented each of the braiding elements as matrices using their Burau representation. These matrices are then multiplied together as the trajectories of the fluid elements braid and we computed the braiding factor $\lambda(t)$ which is the largest eigenvalue of the matrix $M^{(P)}$ defined in (3.4). Other useful invariants could have been computed from the resulting braid (Berger, 2001), but we believe the braiding factor gives the clearest interpretation of mixing in the flow, and thus is the best diagnostic to consider. We also defined a modification of the braiding factor which removes spurious effects on the Burau matrices caused by rotation.

We found that the braiding factor (and hence the braiding exponent) for three particles gave useful information about the mixing properties of the vortex, but only if all three particles are located in the same region of the flow. When this is the case we found a very clear braiding exponent which increased in regions with more chaos. When the three particles were more spread out in the flow we still found a positive braiding exponent as long as at least one particle traced out a chaotic trajectory. Usually in this case the regions of exponential growth in $\lambda(t)$ are interspersed with regions of linear growth, this suggests that mixing is occurring and then stopping on a regular basis. However, for the flow examples in this paper we knew this was not the case and thus this is a limitation of the 3-braid braiding factor. We found that this limitation can be overcome by considering more fluid particles that span a larger area of the flow field. By using the 50-braid braiding factor we found more consistent results, and if we know information about the flow field, then we can cluster the points 
around the region where mixing is expected to take place. One problem that comes from this multiple particle approach is the uncertainty of how many particles should be used. Fifty particles gave a good, approximately straight line (for example see result 4 in figure 13(a)) but this took approximately 16 hours of CPU time, whereas 25 particles takes only a quarter of this time due to the $p^{2}$ nature of the algorithm, where $p$ is the number of particles. Therefore a balance needs to be achieved between time taken to compute the braiding factor and the number of particles used in the computation. The other issue comes from the slow increase in the braiding factor as the number of particles is increased. This slow drift means that it will be difficult to give a definitive braiding exponent for a flow, but as long as $p$ is large enough then the results should be consistent.

Using this 50-braid braiding factor we examined the mixing properties of a multiple frequency strain field which exhibits resonance overlap (akin to a random forcing) for both the kinematic flow model and dynamical flow model. The results showed that braiding occurred faster in the dynamical flow model (i.e. has a larger maximum rate), while the total braiding was slightly more in the kinematic flow model. This was because, the vortex in the dynamical flow model spreads more rapidly, due to the enhanced mixing and therefore there was less time where the vortex contained complex Lagrangian motion.

We conclude that using the braiding diagnostic is useful for determining the amount of mixing within a flow field and we believe that it is beneficial over diagnostics such as Lyapunov exponents because information of the flow field and its derivatives is not required. Although, it is important to consider a sufficient amount of particles in the flow to give a good global picture of the mixing. Further investigation of other invariants of the braid itself is now required to determine if they can improve the understanding of dynamical fluid problems.

\section{Acknowledgments}

The authors would like to thank Andrew Gilbert for his useful comments and suggestions in the production of this article. This work was partially supported by the EPSRC grants EP/D032202/1 (University of Exeter), EP/F069855/1 (University of Brighton) and EP/G000034/1 (University of Keele).

\section{References}

Aref, H. (1984). Stirring by chaotic advection. J. Fluid Mech., 143, 1-21.

Bassom, A. P. and Gilbert, A. D. (1999). The spiral wind-up and dissipation 
of vorticity and a passive scalar in a strained planar vortex. J. Fluid Mech., 398, 245-270.

Berger, M. A. (2001). Topological invariants in braid theory. Lett. Math. Phys., 55(3), 181-192.

Binder, B. J. and Cox, S. M. (2008). A mixer design for the pigtail braid. Fluid Dynam. Res., 40(1), 34-44.

Boyland, P., Aref, H., and Stremler, M. A. (2000). Topological fluid mechanics of stirring. J. Fluid Mechanics, 403, 277-304.

Boyland, P., Stremler, M., and Aref, H. (2003). Topological fluid mechanics of point vortex motions. Phys. D, 175(1-2), 69-95.

Burau, W. (1936). Über zopfgruppen und gleichsinnig verdrillte verkettungen. Abh. Math. Sem. Hanischen Univ., 11, 171-178.

Davis (2004). WOCE subsurface float data assembly centre, http://wfdac. whoi.edu.

Finn, M. D., Cox, S. M., and Byrne, H. M. (2003). Topological chaos in inviscid and viscous mixers. J. Fluid Mech., 493, 345-361.

Jones, V. F. R. (1987). Hecke algebras and a polynomial invariant for knots. Ann. Math, 126, 335-388.

Kin, E. and Sakajo, T. (2005). Efficient topological chaos embedded in the blinking vortex system. Chaos, 15(2), 023111, 9.

Le Dizès, S. (2000). Non-axisymmetric vortices in two-dimensional flows. $J$. Fluid Mech., 406, 175-198.

Lichtenberg, A. J. and Lieberman, M. A. (1992). Regular and Chaotic Dynamics, 2nd ed. Springer (New York).

Moussafir, J.-O. (2006). On computing the entropy of braids. Funct. Anal. Other Math., 1(1), 37-46.

Murasugi, K. (1996). Knot theory and its applications. Birkhäuser (Boston).

Pierrehumbert, R. T. and Yang, H. (1993). Global chaotic mixing on isentropic surfaces. J. Atmos. Sci., 50, 2462-2480.

Shuckburgh, E. and Haynes, P. H. (2003). Diagnosing transport and mixing using a tracer-based coordinate system. Phys. Fluids, 15, 3342-3357.

Thiffeault, J.-L. (2005). Measuring topological chaos. Phys. Rev. Lett., 94, 084502.

Thiffeault, J.-L. (2010). Braids of entangled particle trajectories. Chaos, 20, 017516.

Thiffeault, J.-L., Finn, M. D., Gouillart, E., and Hall, T. (2008). Topology of chaotic mixing patterns. Chaos, 18(3), 033123, 8.

Turner, M. R. and Gilbert, A. D. (2007). Linear and nonlinear decay of cat's eyes in two-dimensional vortices, and the link to Landau poles. J. Fluid Mech., 593, 255-279.

Turner, M. R. and Gilbert, A. D. (2009). Spreading of two-dimensional axisymmetric vortices exposed to a rotating strain field. J. Fluid Mech., 630, $155-177$.

Turner, M. R., Gilbert, A. D., and Thuburn, J. (2008). Effective diffusion of scalar fields in a chaotic flow. Phys. Fluids, 20(10), 107103-1-14. 
Turner, M. R., Bassom, A. P., and Gilbert, A. D. (2009a). Diffusion and the formation of vorticity staircases in randomly strained two-dimensional vortices. J. Fluid Mech., 638, 49-72.

Turner, M. R., Thuburn, J., and Gilbert, A. D. (2009b). The influence of periodic islands in the flow on a scalar tracer in the presence of a steady source. Phys. Fluids, 21(6), 067103.

Vikhansky, A. (2003). Chaotic advection of finite-size bodies in a cavity flow. Phys. Fluids, 15(7), 1830-1836.

Vikhansky, A. (2004). Simulation of topological chaos in laminar flows. Chaos, 14(1), 14-22.

Wolf, A., Swift, J. B., Swinney, H. L., and Vastano, J. A. (1985). Determining Lyapunov exponents from a time series. Physica D, 16, 285-317. 